\title{
Exploiting NOMA for UAV Communications in Large-Scale Cellular Networks
}

\author{
Tianwei Hou, Student Member, IEEE, Yuanwei Liu, Senior Member, IEEE, \\ Zhengyu Song, Xin Sun, Yue Chen, Senior Member, IEEE,
}

\begin{abstract}
This paper advocates a pair of strategies in non-orthogonal multiple access (NOMA) in unmanned aerial vehicles (UAVs) communications, where multiple UAVs play as new aerial communications platforms for serving terrestrial NOMA users. A new multiple UAVs framework with invoking stochastic geometry technique is proposed, in which a pair of practical strategies are considered: 1) the UAV-centric strategy for offloading actions and 2) the user-centric strategy for providing emergency communications. In order to provide practical insights for the proposed NOMA assisted UAV framework, an imperfect successive interference cancelation (ipSIC) scenario is taken into account. For both UAV-centric strategy and user-centric strategy, we derive new exact expressions for the coverage probability. We also derive new analytical results for orthogonal multiple access (OMA) for providing a benchmark scheme. The derived analytical results in both user-centric strategy and UAV-centric strategy explicitly indicate that the ipSIC coefficient is a dominant component in terms of coverage probability. Numerical results are provided to confirm that i) for both user-centric strategy and UAV-centric strategy, NOMA assisted UAV cellular networks is capable of outperforming OMA by setting power allocation factors and targeted rate properly; and ii) the coverage probability of NOMA assisted UAV cellular framework is affected to a large extent by ipSIC coefficient, target rates and power allocations factors of paired NOMA users.
\end{abstract}

\section{Index Terms}

Non-orthogonal multiple access, stochastic geometry, unmanned aerial vehicles.

This work is supported by the Fundamental Research Funds for the Central Universities under Grant 2019YJS008. Part of this work was submitted to the IEEE Vehicular Technology Conference, Hawaii, USA, Fall 2019 [1].

T. Hou, Z. Song and X. Sun are with the School of Electronic and Information Engineering, Beijing Jiaotong University, Beijing 100044, China (email: 16111019@bjtu.edu.cn, xsun@bjtu.edu.cn, songzy@bjtu.edu.cn).

Y. Liu and Yue Chen are with School of Electronic Engineering and Computer Science, Queen Mary University of London, London E1 4NS, U.K. (e-mail: yuanwei.liu@qmul.ac.uk, yue.chen@qmul.ac.uk). 


\section{INTRODUCTION}

In the past decades, much research effort has been directed towards developing remotely operated unmanned aerial vehicles (UAVs), which stand as a potential candidate of aerial base station (BS) to provide access services to wireless devices located on the ground [2] or in the sky [3]. UAV communications are also an effective approach to provide connectivity during temporary events and after disasters in the remote areas that lack cellular infrastructure [2]. As compared to conventional terrestrial communications, one distinct feature of UAV communication is that the existence of line-of-sight (LoS) is capable of offering stronger small-scale fading between UAVs and ground users because of the high altitude of UAVs, which brings both opportunities and challenges in the design of UAV cellular networks [4]. Due to the limited energy resources on board of a UAV, achieving higher spectrum efficiency and energy efficiency is of paramount importance to reap maximum benefits from UAV based communication networks.

To exploit both the spectrum efficiency and energy efficiency in the next generation wireless networks and beyond, especially in the UAV communication networks, non-orthogonal multiple access (NOMA) is considered to be a promising technique [5], [6]. More specifically, in contrast to the conventional OMA techniques, NOMA is capable of exploiting the available resources more efficiently by opportunistically capitalizing on the users specific channel conditions on both single cell networks and cellular networks [7], [8], and it is capable of serving multiple users at different quality-of-service (QoS) requirements in the same resource block [9]-[11]. To be more clear, NOMA technique sends the composite signal to multiple users simultaneously by power domain multiplexing within the same frequency, time and code block. The basic principles of NOMA techniques rely on the employment of superposition coding (SC) at the transmitter and successive interference cancelation (SIC) techniques at the receiver [5], [12], and hence multiple accessed users can be realized in the power domain via different power levels for users in the same resource block 1 . Therefore, UAV networks can serve multiple users simultaneously by utilizing NOMA techniques for enhancing the achievable spectrum efficiency and energy efficiency.

\footnotetext{
${ }^{1}$ In this paper, we use NOMA to refer to power-domain NOMA for simplicity.
} 


\section{A. Prior Work and Motivation}

Regarding the literature of UAV networks, early research contributions have studied the performance of single UAV or multiple UAVs networks. Mozaffari et al. [3] proposed a UAV assisted underlaid D2D network with LoS probability, which depends on the height of the UAV, the horizontal distance between the UAV and users, the carrier frequency and type of environment. In the case that LoS exists, a fixed LoS coefficient, e.g., an extra $20 \mathrm{~dB}$ attenuation, is the dominant component of small-scale fading channels. Note that the proposed model in [3], [4] is a practical model for implementation. For mathematically tractable, the distinctive channel characteristics for UAV networks were investigated in [13], where different types of small-scale fading channels, i.e., Loo model, Rayleigh model, Nakagami-m model, Rician model and Werbull model, were summarized to demonstrate the channel propagation of UAV networks. The air-to-air channel characterization in [14], studied the influence of the altitude-dependent Rician K factor. This work indicated that the impact of the ground reflected multi-path fading reduces with increasing UAV altitude. Jiang et al. [15] proposed a UAV assisted ground-to-air network, where Rician channels are used for evaluating strong LoS components between UAV and ground users. It is also worth noting that Rayleigh fading channel, which is a well-known model in scattering environment, can be also used to model the UAV channel characteristics in the case of large elevation angles in the mixed-urban environment. Chetlur et al. [16] proposed a downlink UAV network over Nakagami- $m$ fading channels, where UAVs are distributed in a finite 3-D network. An uniform binomial point process was invoked to model the proposed network. Generally speaking, Nakagami- $m$ distribution and Rician distribution are used to approximate the fluctuations in the fading channel with LoS propagations. It is also worth noting that the fading parameter of Nakagami- $m$ fading $m=\frac{(K+1)^{2}}{2 K+1}$, the distribution of Nakagami- $m$ is approximately Rician fading with parameter $K$ [17, eq. (3.38)]. Zhang et al. [18] proposed two possible paradigms for UAV assisted cellular communications, namely, cellular-enabled UAV communication and UAVassisted cellular communication. The trajectory of the UAV was optimized under connectivityconstrained. Lyu et al. [19] proposed a UAV assisted cellular hotspot scenario, where UAV flies cyclically along the cell edge for offloading actions. In order to improve the spectrum efficiency and energy efficiency of UAV communications, new research on UAV under emerging next generation network architectures is needed.

Recently, the use of NOMA in wireless communication has attracted great interest in single 
cell or cellular networks [11], [20], [21]. Ding et al. [22] evaluated the performance of NOMA enhanced single cell networks with randomly deployed users, where order statistics and stochastic geometry tools were invoked to evaluate the performance of paired NOMA users. Some application scenarios of NOMA have been investigated in the previous literature. More particularly, Liu et al. [23] proposed an innovative model of cooperative NOMA with simultaneous wireless information and power transfer (SWIPT), where a NOMA cluster consists of two NOMA users, one that is located in a small disk and the other is in a ring with a larger external radius. Ding et al. [7] evaluated the performance of NOMA with fixed power allocation (F-NOMA) and cognitive radio inspired NOMA (CR-NOMA), and the user pairing strategies were carefully discussed. The analytical results show that it is more preferable to pair users whose channel gains are more distinctive to improve the diversity order in F-NOMA, whereas CR-NOMA prefers to pair the users with the best channel conditions. Recently, an imperfect SIC scenario has attracted great interest. Due to the fact that SIC techniques are deployed at the receivers, the residues of the multiplexed signal detected by SIC technique cannot be ignored [24]. Once an error occurs for carrying out SIC at the user with better channel gain, the NOMA systems will suffer from the residual interference signal. Hence it is significant to examine the detrimental impacts of imperfect SIC for NOMA system. Hou et al. [25] evaluated the outage performance of NOMA downlink transmission in both $\operatorname{LoS}$ and NLoS scenarios. A potential future research direction for NOMA, called Rate-Splitting multiple access, has been proposed by Mao et al. [26]. The analytical results in [26] demonstrated that RSMA can outperform SDMA and NOMA in the multi-antenna system and comes with a lower complexity than NOMA. RSMA assisted multicell networks and multi-antenna assisted RSMA were also proposed in [27]. The results derived concluded that RSMA can provide rate, robustness and QoS enhancements over SDMA and NOMA. With the goal of enhancing the physical layer security of NOMA networks, Liu et al. [28] proposed a NOMA assisted physical layer security framework in large-scale networks, where both single antenna and multiple antenna aided transmission scenarios were considered.

In UAV-enabled wireless communications, the total UAV energy is limited, which includes propulsion energy and communication related energy [29]. Therefore, integrating UAVs and NOMA into cellular networks is considered to be a promising technique to significantly enhance the performance of terrestrial users in the next generation wireless system and beyond, where the energy efficiency and spectrum efficiency can be greatly enhanced in downlink transmission to minimize communication related energy [30]. A general introduction of UAV communications 
has been proposed by Liu et al. [31]. Three case studies, i.e., performance evaluation, joint trajectory design, and machine learning assisted UAV deployment [32], were carried out in order to better understand NOMA enabled UAV networks. Some challenges were concluded for future research directions. Zhao et al. [33] proposed a UAV-assisted NOMA network, where UAV and BS are cooperated to provide access services to ground users simultaneously. The trajectory of UAV and precoding matrix of BS were jointly optimized. Nguyen et al. [34] proposed a cooperative multi-UAV network, where a fixed number of UAVs are used as flying relays in wireless backhaul networks, and the small-scale fading follows Rician distributions. Hou et al. [35] proposed a multiple-input multiple-output (MIMO)-NOMA assisted UAV network, where the closed-form expressions of outage performance and ergodic rate were evaluated in the downlink scenario. A NOMA assisted uplink scenario of UAV assisted cellular communication was proposed by Mei et al. [36], where two special cases, i.e., egoistic and altruistic transmission strategies of the UAV, were considered to derive the optimized solutions. Liu et al. [37] proposed a MIMO-NOMA assisted UAV network for uplink transmission, where the cellular-connected UAV communication with air-to-ground interference was investigated by utilizing multi-beam techniques. Han et al. [38] proposed a UAV assisted millimeter-wave air-to-everything networks, where aerial access points provide access services to users located on the ground, air, and tower. The buildings were modeled as a Boolean line-segment process with the fixed height.

The previous contributions [31], [34]-[39] mainly consider NOMA in single UAV cell or NOMA assisted uplink transmission, and thus do not account for NOMA assisted downlink transmission in UAV assisted cellular networks. The research contributions in terms of conducting on multi-UAV aided NOMA networks are still in their infancy, particularly with the focus of potential association strategies. NOMA enhanced UAV networks design poses three additional challenges: i) NOMA technology brings additional intra-cell interference from the connected UAV to the served users; ii) UAV communication requires different fading channels to evaluate the channel gain of LoS/NLoS propagation. iii) the user association policy requires to be reconsidered in NOMA assisted UAV networks. In this article, aiming at tackling the aforementioned issues, by proposing two potential association strategies, namely UAV-centric strategy and user-centric strategy, for intelligently investigating the effect of NOMA assisted UAV network performance is desired. The motivation of proposing two strategies is that the user-centric strategy is a promising solution for providing access services after disasters in the remote areas, where all of terrestrial users located in the Voronoi cell can be served by UAVs. 
On the contrary, the UAV-centric strategy can be perfectly deployed in the dense networks, i.e., concerts or football matches, to provide supplementary access services for offloading actions, where terrestrial users are located in a regular disc. Note that one other non-negligible difference between the two strategies is that user association is decided by individual user or UAV for the user-centric strategy or the UAV-centric strategy, respectively. Stochastic geometry tools are invoked to provide the mathematical paradigm to model the spatial randomness of both UAVs and users in UAV cellular networks. In contrast to the conventional terrestrial communication structure, where the locations of BSs are fixed, stochastic geometry is more suitable for analyzing the average performance of the mobility and flexibility of the UAV networks.

\section{B. Contributions}

In contract to most existing research contributions in context of UAV communications [31], [34]-[39], we consider a multi-cell set-up in this paper. We propose two new NOMA assisted UAV cellular strategies, namely user-centric strategy and UAV-centric strategy. Based on the proposed strategies, the primary theoretical contributions can be summarized as follows:

- We develop two potential association strategies to address the impact of NOMA on the UAV communications, namely user-centric strategy and UAV-centric strategy, where stochastic geometry approaches are invoked to model the locations of both UAVs and users.

- For the user-centric strategy: we derive the exact analytical expressions of a typical user in the NOMA enhanced user-centric strategy in terms of coverage probability. Additionally, we derive the exact expressions in terms of coverage probability for the OMA assisted user-centric strategy. Our analytical results illustrate that the distance of the fixed user has effect on the coverage probability of the typical user. Furthermore, for the case of poor SIC quality, a hybrid NOMA/OMA assisted UAV framework may be a good solution.

- For the UAV-centric strategy: we derive the exact analytical expressions of paired NOMA users in the NOMA enhanced UAV-centric strategy in terms of coverage probability. The exact expressions in terms of coverage probability for the OMA case are derived. Our analytical results indicates that the UAV-centric strategy is more susceptible to ipSIC factor than the user-centric strategy.

- Simulation results confirm our analysis, and illustrate that by setting power allocation factors and targeted rate properly, NOMA assisted UAV cellular frameworks has superior performance over OMA assisted UAV cellular frameworks in terms of coverage probability, 


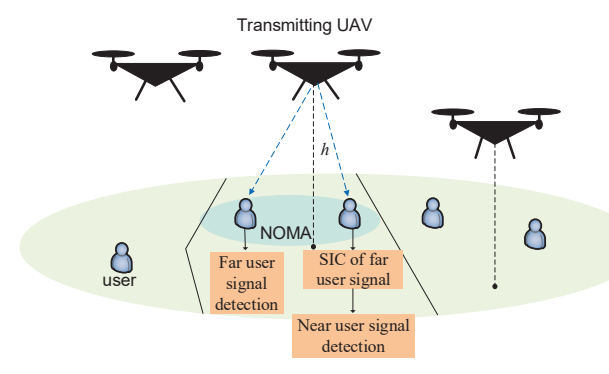

(a) Illustration of the system model.

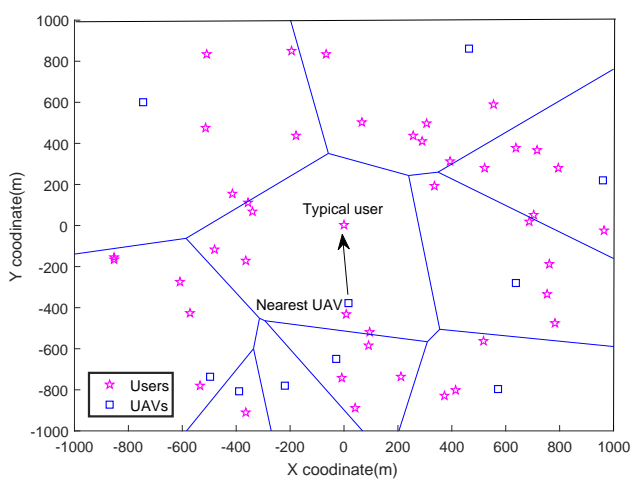

(b) Top view of the user-centric strategy cellular networks.

Fig. 1: Illustration of NOMA assisted user-centric strategy model.

which demonstrates the benefits of the proposed strategies. Our analytical results also illustrate that the coverage probability can be greatly enhanced by LoS links.

\section{Organization}

The rest of the paper is organized as follows. In Section II, the NOMA assisted user-centric strategy is investigated for UAV cellular frameworks, where the UAV provides access services to all the users. In Section III, the NOMA assisted UAV-centric strategy is investigated, where the UAV only provides access services to the restricted areas. Our numerical results are demonstrated in Section IV for verifying our analysis, which is followed by the conclusion in Section V.

\section{UsER-CENTRIC STRATEGY FOR EMERGENCy COMMUNICATIONS}

We first focus our attention on a scenario, where all the terrestrial users are needed to be served equally for emergency communications, e.g., after disasters, in the remote areas or in the rural areas [40]. Motivated by this purpose, we propose the user-centric strategy for providing emergency access services to all the terrestrial users.

Focusing on downlink transmission scenarios, we consider the user-centric strategy as shown in Fig. 1(a), In this article, the UAV equipped with a single antenna communicates with multiple users equipped with a single antenna each. In the user-centric strategy, the locations of terrestrial users are totally random for emergency services, and there are no further information for UAVs to properly organize their trajectory. In order to serve all the terrestrial users equally, multiple UAVs should be distributed uniformly, which conforms the definition of homogeneous poisson point process (HPPP). Thereby, the UAVs are distributed according to a HPPP $\Psi$ with density $\lambda$. 
For the simplicity of theoretical analysis, as shown in Fig. 1(b), an user is located at the original point in the user-centric strategy, which becomes the typical user. The user-centric strategy is a useful model for the large-scale networks, i.e., rural area, where users are uniformly located in the Voronoi cell according to a HPPP $\Phi_{u}$ with density $\lambda_{u}$. It is worth mentioning that in the case that the density of user $\lambda_{u}$ is low, the user-centric performs much better than the UAV-centric strategy.

Without loss of generality, we consider that there is one user, namely fixed user, is already connected to the UAV in the previous round of user association process 2 . For simplicity, we assume that the horizontal distances between the fixed user and the connected UAV is $r_{k}$, which can be any arbitrary values, and the horizontal distance between the typical user and the connected UAV is random, denoted by $r$. In the user-centric strategy, we consider that two users, fixed user and typical user, are paired to perform NOMA technique, where paired NOMA users share the same frequency, time and code resource blocks.

\section{A. Channel Model}

Consider the use of a composite channel model with two parts, large-scale fading and smallscale fading. It is assumed that the horizontal distance $r$ and the height of the UAV $h$ are independent and identically distributed (i.i.d.). In this article, large-scale fading represents the path loss between the UAV and users. 3 .

In order to better illustrate the LoS propagation between the UAV and user, the small-scale fading is defined by Nakagami- $m$ fading, and the probability density function (PDF) can be expressed as

$$
f(x)=\frac{m^{m} x^{m-1}}{\Gamma(m)} e^{-m x},
$$

where $m$ denotes the fading parameter, and $\Gamma(m)$ denotes Gamma function. Note that $\Gamma(m)=$ $(m-1)$ ! when $m$ is an integer. The serving distance between the connected UAV to the typical user can be written as

$$
r_{t}=\sqrt{h^{2}+r^{2}}
$$

\footnotetext{
${ }^{2}$ In practice, multiple users are connected to the transmitter (UAV) one by one.

${ }^{3}$ A log-normal distributed random variable for shadowing on both the desired and interference signals was considered in [41], which indicates that Lognormal shadowing on both the desired and interfering signals does not significantly affect the accuracy of numerical analysis. Thus, we neglect it in this article for simplicity.
} 
where $r$ is the nearest horizontal distance allowed between a typical user and its connected UAV.

In order to avoid infinite received power, it is assumed that the height of the UAV is greater than $1 \mathrm{~m}$ to simplify the analytical results. Therefore, the large-scale fading can be expressed as

$$
L_{t}=r_{t}^{-\alpha}
$$

where $\alpha$ denotes the path loss exponent between the typical user and the connected UAV. Thus, the received power from the associated UAV for the user at origin is given by

$$
P_{t}=P_{u} L_{t}\left|h_{t}\right|^{2}
$$

where $P_{u}$ denotes the transmit power of the UAV, and $h_{t}$ denotes the channel coefficients for the typical user and its associated UAV.

In downlink transmission, paired NOMA users also detect interference from neighboring UAVs. Therefore, the co-channel interference $I$ can be further expressed as follows:

$$
I \triangleq \sum_{j \in \Psi, d_{j}>r_{t}}\left|g_{j}\right|^{2} P_{u} d_{j}^{-\alpha_{I}}
$$

where $d_{j}$ and $\left|g_{j}\right|^{2}$ denote the distance and the small-scale fading between the user and the $j$-th interfering UAV, $\alpha_{I}$ denotes the path loss exponent between interfering UAV and the typical user.

Besides, in practical wireless communication systems, obtaining the channel state information (CSI) at the transmitter or receiver is not a trivial problem, which requires the classic pilot-based training process. Therefore, in order to provide more engineering insights, it is assumed that the CSI of UAVs is partly known at the typical user, where only distance information between UAVs and the typical user is required. The signal-to-interference-plus-noise ratio (SINR) of the user-centric strategy will be derived in the following subsection.

\section{B. SINR Analysis}

For the user-centric strategy, since the distance of typical user and its associated UAV is not pre-determined. Therefore, focusing on the typical user, there are two potential cases, namely far user case and near user case, where 1) far user case, i.e., $r>r_{k}$; and 2) near user case, i.e., $r<r_{k}$. We then turn our attention on the SINR analysis of two potential cases.

(1) Far user case: 
For the far user case, where the serving distance of the typical user is greater than that of the fixed user, the typical user treats the signal from the fixed user as noise, and thus the SINR can be expressed as

$$
S I N R_{t, f a r}=\frac{\left|h_{t}\right|^{2} r_{t}^{-\alpha} P_{u} \alpha_{v}^{2}}{\sigma^{2}+\left|h_{t}\right|^{2} r_{t}^{-\alpha} P_{u} \alpha_{w}^{2}+\sum_{j \in \Psi, d_{j}>r_{t}}\left|g_{j}\right|^{2} P_{u} d_{j}^{-\alpha_{I}}}
$$

where $\sigma^{2}$ denotes the additive white Gaussian noise (AWGN) power, $\alpha_{v}^{2}$ and $\alpha_{w}^{2}$ denote the power allocation factors for the far user and the near user, respectively. Note that $\alpha_{v}^{2}+\alpha_{w}^{2}=1$ in NOMA communication.

For the far user case, SIC technique is deployed at the fixed user, thereby the fixed user needs to decode the information from the typical user with the following SINR

$$
S I N R_{f \rightarrow t, f a r}=\frac{\left|h_{f}\right|^{2} R_{k}^{-\alpha} P_{u} \alpha_{v}^{2}}{\sigma^{2}+\left|h_{f}\right|^{2} R_{k}^{-\alpha} P_{u} \alpha_{w}^{2}+\sum_{j \in \Psi, d_{j}>r_{t}}\left|g_{j}\right|^{2} P_{u} d_{j}^{-\alpha_{I}}},
$$

where $R_{k}=\sqrt{r_{k}^{2}+h^{2}}$, and $h_{f}$ denotes the channel coefficients for the fixed user.

Once it is decoded successfully, the fixed user will decode its own signal with imperfect SIC coefficient, and the SINR can be expressed as

$$
S I N R_{f, f a r}=\frac{\left|h_{f}\right|^{2} R_{k}^{-\alpha} P_{u} \alpha_{w}^{2}}{\sigma^{2}+\beta\left|h_{f}\right|^{2} R_{k}^{-\alpha} P_{u} \alpha_{v}^{2}+\sum_{j \in \Psi, d_{j}>r_{t}}\left|g_{j}\right|^{2} P_{u} d_{j}^{-\alpha_{I}}},
$$

where $\beta$ denotes the imperfect SIC coefficient. Since in practice that SIC is not perfect, a fraction $0<\beta<1$ is considered in our model for the user with better channel gain. On the one hand, $\beta=0$ when perfect SIC is assumed, and the near user can perfectly decode the signal intended for the far user. On the other hand, when SIC is failed or there is no corresponding SIC, $\beta=1$.

(2) Near user case:

For the near user case, when the typical user has smaller serving distance to the UAV than that of the fixed user, the signal of the typical user can be treated as noise at the fixed user, and thus the SINR of the fixed user can be expressed as

$$
S I N R_{f, \text { near }}=\frac{\left|h_{f}\right|^{2} R_{k}^{-\alpha} P_{u} \alpha_{v}^{2}}{\sigma^{2}+\left|h_{f}\right|^{2} R_{k}^{-\alpha} P_{u} \alpha_{w}^{2}+\sum_{j \in \Psi, d_{j}>r_{t}}\left|g_{j}\right|^{2} P_{u} d_{j}^{-\alpha_{I}}} .
$$

The SIC technique can be deployed at the typical user for decoding the signal from the fixed 
user, and the SINR at the typical user for the near user case can be expressed as

$$
S I N R_{t \rightarrow f, \text { near }}=\frac{\left|h_{t}\right|^{2} r_{t}^{-\alpha} P_{u} \alpha_{v}^{2}}{\sigma^{2}+\left|h_{t}\right|^{2} r_{t}^{-\alpha} P_{u} \alpha_{w}^{2}+\sum_{j \in \Psi, d_{j}>r_{t}}\left|g_{j}\right|^{2} P_{u} d_{j}^{-\alpha_{I}}}
$$

Once the typical user decodes the information from the fixed user successfully, the typical user can decode its own signal with the SINR

$$
S I N R_{t, \text { near }}=\frac{\left|h_{t}\right|^{2} r_{t}^{-\alpha} P_{u} \alpha_{w}^{2}}{\sigma^{2}+\beta\left|h_{t}\right|^{2} r_{t}^{-\alpha} P_{u} \alpha_{v}^{2}+\sum_{j \in \Psi, d_{j}>r_{t}}\left|g_{j}\right|^{2} P_{u} d_{j}^{-\alpha_{I}}}
$$

\section{Coverage Probability of the User-centric Strategy}

In the networks considered, we first focus on analyzing the PDF of user distance distributions for paired NOMA users, which will be used for both user-centric strategy and UAV-centric strategy.

Lemma 1. The UAVs are distributed according to a HPPP with density $\lambda$. It is assumed that the typical user is located at the origin of the disc in the user-centric strategy, or the typical UAV is located at the origin of the disc in the UAV-centric strategy, which is under expectation over HPPP. Thus, the horizontal distance $r$ between the origin and UAVs, follows the distribution

$$
f_{r}(r)=2 \pi \lambda r e^{-\pi \lambda r^{2}}, r \geq 0
$$

Then, we focus on analyzing the user-centric strategy of the proposed framework in order to increase the system fairness. In the user-centric strategy, the user association is based on connecting the nearest UAV to the typical user. As such, the first step is to derive the Laplace transform of interference for the typical user.

Lemma 2. For the user-centric strategy, and based on the characteristic of stochastic geometry, the interference received at both typical user and fixed user can be recognized as the same. Therefore, the Laplace transform of interference distribution for the paired NOMA users is given by

$$
\mathcal{L}_{t}(s)=\exp \left(-\frac{2 \pi \lambda}{\alpha_{I}} \sum_{i=1}^{m_{I}}\left(\begin{array}{c}
m_{I} \\
i
\end{array}\right)\left(\frac{s P_{u}}{m_{I}}\right)^{\delta_{I}}(-1)^{\delta_{I}-i} B\left(\frac{-s P_{u}}{m_{I} r_{t}^{\alpha_{I}}} ; i-\delta_{I}, 1-m_{I}\right)\right),
$$


where $\delta_{I}=\frac{2}{\alpha_{I}}, m_{I}$ denotes the fading parameters between a typical user and interfering UAVs, and $B(;)$ denotes incomplete Beta function.

Proof. Please refer to Appendix A.

In the case of large-scale networks, the existence of LoS propagations between interfering UAVs at infinity and users is not reasonable. Thus, the minimum received power of inter-cell interference for cellular UAV networks is worth estimating, where the fading parameters between ground users and interfering UAVs equal to one. It is also assumed that the path loss exponent $\alpha_{I}=4$ because that path loss exponent is normally in the range of 2 to 4 , where 2 is for propagation in free space, 4 is for relatively lossy environments and in the case of full specular reflection from the earth surface.

Corollary 1. For the special case that the small scale fading channels between interfering UAVs and users follow Rayleigh fading, thereby $m_{I}=1$ and $\alpha_{I}=4$ for the user-centric strategy, the Laplace transform of interference distribution for the both paired NOMA users can be transformed into

$$
\begin{aligned}
\mathcal{L}_{t}(s) & \stackrel{(a)}{=} \exp \left(-\frac{2 \pi \lambda P_{u} r_{t}^{2-\alpha_{I}}}{\alpha_{I}\left(1-\delta_{I}\right)}{ }_{2} F_{1}\left(1,1-\delta_{I} ; 2-\delta_{I} ;-s P_{u} r_{t}^{-\alpha_{I}}\right)\right) \\
& \stackrel{(b)}{=} \exp \left(-\pi \lambda \sqrt{s P_{u}} \tan ^{-1}\left(\frac{\sqrt{s P_{u}}}{r_{t}^{2}}\right)\right),
\end{aligned}
$$

where $(a)$ is resulted from applying $m_{I}=1,(b)$ is obtained by substituting $\alpha_{I}=4$, and ${ }_{2} F_{1}(; ;)$ denotes Gauss hypergeometric function.

Then, we focus on the coverage behavior of the user-centric strategy. The fixed power allocation strategy is deployed at the UAV, where the power allocation factors $\alpha_{w}^{2}$ and $\alpha_{v}^{2}$ are constant during transmission. It is assumed that the target rates of the typical user and the fixed user are $R_{t}$ and $R_{f}$, respectively. Based on SINR analysis in (6), (10) and (11), the coverage probability of the typical user can be expressed as follows:

$$
\begin{aligned}
& P_{t}(r)=P_{t, \text { near }}(r) P\left(r<r_{k}\right)+P_{t, \text { far }}(r) P\left(r>r_{k}\right) \\
& =\operatorname{Pr}\left(S I N R_{t \rightarrow f, \text { near }}>\varepsilon_{f}, S I N R_{t, \text { near }}>\varepsilon_{t}\right) \operatorname{Pr}\left(r<r_{k}\right)+\operatorname{Pr}\left(S I N R_{t, \text { far }}>\varepsilon_{t}\right) \operatorname{Pr}\left(r>r_{k}\right),
\end{aligned}
$$

where $\varepsilon_{t}=2^{R_{t}}-1, \varepsilon_{f}=2^{R_{f}}-1, P_{t, \text { near }}(r)$ and $P_{t, f a r}(r)$ denote the coverage probability of the typical user for the near user case and the far user case, respectively. $P\left(r>r_{k}\right)$ and $P\left(r<r_{k}\right)$ 
denote the probability of far user case and near user case, respectively. Therefore, the coverage probability of the typical user for the near user case and far user case is given in following two Lemmas.

Lemma 3. The coverage probability conditioned on the serving distance of a typical user for the near user case in the user-centric strategy is expressed in closed-form as

$$
\begin{aligned}
P_{t, \text { near }}(r) & =\sum_{n=0}^{m-1} \sum_{p=0}^{n}\left(\begin{array}{l}
n \\
p
\end{array}\right) \frac{(-1)^{n}}{n !} \Lambda_{4}^{n} \Lambda_{5}^{n} \exp \left(-m M_{t *} \sigma^{2} r_{t}^{\alpha}-\Lambda_{3} r_{t}^{2+\left(\alpha-\alpha_{I}\right)(i+a)}\right) \\
& \times r_{t}^{\alpha(1-j) q_{j}+\left(2+\left(\alpha-\alpha_{I}\right)(i+a)-\alpha b\right) q_{b}+\alpha n},
\end{aligned}
$$

where $M_{t}^{n}=\frac{\varepsilon_{t}}{P_{u}\left(\alpha_{w}^{2}-\beta \varepsilon_{t} \alpha_{v}^{2}\right)}, M_{t \rightarrow f}=\frac{\varepsilon_{f}}{P_{u}\left(\alpha_{v}^{2}-\varepsilon_{f} \alpha_{w}^{2}\right)}, M_{t *}=\max \left\{M_{t}^{n}, M_{t \rightarrow f}\right\}, r_{t}=\sqrt{r^{2}+h^{2}}$,

$\Lambda_{3}=\frac{2 \pi m \lambda}{\alpha_{I}} \sum_{a=0}^{\infty} \frac{\left(m_{I}\right)_{a}}{a !\left(i-\delta_{I}+a\right)} \sum_{i=1}^{m_{I}}\left(\begin{array}{c}m_{I} \\ i\end{array}\right)\left(\frac{M_{t *} P_{u}}{m_{I}}\right)^{i+a}(-1)^{a}, \Lambda_{4}^{n}=\sum p ! \prod_{j=1}^{p} \frac{\left(\left(-m M_{t *} \sigma^{2}\right) \prod_{k=0}^{j-1}(1-k)\right)^{q_{j}}}{q_{j} !(j !)^{q_{j}}}$, and $\Lambda_{5}^{n}=\sum(n-p) ! \prod_{b=1}^{n-p} \frac{\left(\left(-\Lambda_{3}\right) \prod_{k=0}^{b-1}\left(\delta_{I}-k\right)\right)^{q_{b}}}{q_{b} !(b !)^{q_{b}}}$.

Proof. Please refer to Appendix B.

For the far user case, note that decoding will succeed if the typical user can decode its own message by treating the signal from the fixed user as noise. The coverage probability conditioned on the serving distance of a typical user for the far user case is calculated in the following Lemma.

Lemma 4. The coverage probability conditioned on the serving distance of a typical user for the far user case in the user-centric strategy is expressed in closed-form as

$$
\begin{aligned}
P_{t, f a r}(r) & =\sum_{n=0}^{m-1} \sum_{p=0}^{n}\left(\begin{array}{l}
n \\
p
\end{array}\right) \frac{(-1)^{n}}{n !} \Lambda_{4}^{f} \Lambda_{5}^{f} \exp \left(-m M_{t}^{f} \sigma^{2} r_{t}^{\alpha}-\Lambda_{3}^{f} r_{t}^{2+\left(\alpha-\alpha_{I}\right)(i+a)}\right) \\
& \times r_{t}^{\alpha(1-j) q_{j}+\left(2+\left(\alpha-\alpha_{I}\right)(i+a)-\alpha b\right) q_{b}+\alpha n},
\end{aligned}
$$

where $M_{t}^{f}=\frac{\varepsilon_{t}}{P_{u}\left(\alpha_{v}^{2}-\varepsilon_{t} \alpha_{w}^{2}\right)}, \Lambda_{3}^{f}=\frac{2 \pi m \lambda}{\alpha_{I}} \sum_{a=0}^{\infty} \frac{\left(m_{I}\right)_{a}}{a !\left(i-\delta_{I}+a\right)} \sum_{i=1}^{m_{I}}\left(\begin{array}{c}m_{I} \\ i\end{array}\right)\left(\frac{M_{t}^{f} P_{u}}{m_{I}}\right)^{i+a}(-1)^{a}$,

$\Lambda_{4}^{f}=\sum p ! \prod_{j=1}^{p} \frac{\left(\left(-m M_{t}^{f} \sigma^{2}\right) \prod_{k=0}^{j-1}(1-k)\right)^{q_{j}}}{q_{j} !(j !)^{q_{j}}}$, and $\Lambda_{5}^{f}=\sum(n-p) ! \prod_{b=1}^{n-p} \frac{\left(\left(-\Lambda_{3}^{f}\right) \prod_{k=0}^{b-1}(\delta-k)\right)^{q_{b}}}{q_{b} !(b !)^{q_{b}}}$.

Proof. Based on the SINR analysis in (6), and following the similar procedure in Appendix B, with interchanging $M_{t *}$ with $M_{t}^{f}$, we can obtain the desired result in (17). Thus, the proof is complete. 
Remark 1. The derived results in (16) and (17) demonstrate that the coverage probability of a typical user is determined by imperfect SIC coefficient, the target rate of itself, fading parameter $m$ of the small scale fading channels and the distance of the fixed user served by the same UAV.

Remark 2. Inappropriate power allocation such as, $\alpha_{v}^{2}-\varepsilon_{t} \alpha_{w}^{2}<0$ and $\alpha_{w}^{2}-\beta \varepsilon_{t} \alpha_{v}^{2}<0$, will lead to the coverage probability always being zero.

Based on Lemma 3 and Lemma 4, the coverage probability of the typical user in the usercentric strategy can be calculated in the following Theorem.

Theorem 1. The exact expression of the coverage probability for the typical user is expressed as

$$
P_{t}=\int_{0}^{r_{k}} P_{t, \text { near }}(r) f_{r}(r) d r+\int_{r_{k}}^{\infty} P_{t, f a r}(r) f_{r}(r) d r
$$

where $P_{t, \text { near }}(r)$ is given in (16), $P_{t, f a r}(r)$ is given in (17), and $f(r)$ is given in (12).

Remark 3. Based on the result in (18), the coverage probability of the typical user is dependent on the distance of the fixed user in the user-centric strategy.

In order to provide more insights for UAV assisted cellular networks, the coverage probability of the typical user is also derived in the OMA assisted UAV cellular networks, i.e., TDMA. The typical user and fixed user follow the same distance distributions and small-scale fading channels in the OMA assisted cellular UAV networks. The OMA benchmark adopted in this article is that by dividing the two users in equal time/frequency slots.

Corollary 2. The coverage probability conditioned on the serving distance of a typical user for the OMA assisted UAV cellular networks in the user-centric strategy is expressed in closed-form as

$$
\begin{aligned}
P_{c o v, t, o}(r) & =\sum_{n=0}^{m-1} \sum_{p=0}^{n}\left(\begin{array}{l}
n \\
p
\end{array}\right) \frac{(-1)^{n}}{n !} \Lambda_{4}^{o} \Lambda_{5}^{o} \exp \left(-m M_{t}^{o} \sigma^{2} r_{t}^{\alpha}-\Lambda_{3}^{o} r_{t}^{2+\left(\alpha-\alpha_{I}\right)(i+a)}\right) \\
& \times r_{t}^{\alpha(1-j) q_{j}+\left(2+\left(\alpha-\alpha_{I}\right)(i+a)-\alpha b\right) q_{b}+\alpha n},
\end{aligned}
$$

where $M_{t}^{o}=\frac{\varepsilon_{t}^{o}}{P_{u}}, \varepsilon_{t}^{o}=2^{2 R_{t}}-1, \Lambda_{3}^{o}=\frac{2 \pi m \lambda}{\alpha_{I}} \sum_{a=0}^{\infty} \frac{\left(m_{I}\right)_{a}}{a !\left(i-\delta_{I}+a\right)} \sum_{i=1}^{m_{I}}\left(\begin{array}{c}m_{I} \\ i\end{array}\right)\left(\frac{M_{t}^{o} P_{u}}{m_{I}}\right)^{i+a}(-1)^{a}$,

$$
\Lambda_{4}^{o}=\sum p ! \prod_{j=1}^{p} \frac{\left(\left(-m M_{t}^{o} \sigma^{2}\right) \prod_{k=0}^{j-1}(1-k)\right)^{q_{j}}}{q_{j} !(j !)^{q_{j}}} \text {, and } \Lambda_{5}^{o}=\sum(n-p) ! \prod_{b=1}^{n-p} \frac{\left(\left(-\Lambda_{3}\right) \prod_{k=0}^{b-1}\left(\delta_{I}-k\right)\right)^{q_{b}}}{q_{b} !(b !)^{q_{b}}} \text {. }
$$




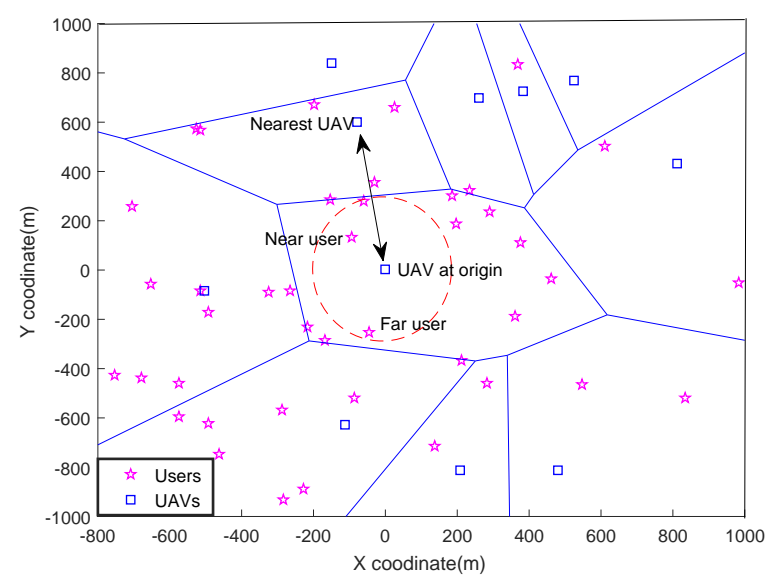

Fig. 2: Top view of the UAV-centric strategy cellular networks.

Proof. Following the similar procedure in Appendix B, with interchanging $M_{t}^{f}$ with $M_{t}^{o}$, we can obtain the desired result in (19). Thus, the proof is complete.

\section{UAV-CENTRIC StRATEgy FOR OfFlOAding ACtions}

In conventional BS communications, the BSs are distributed in order to cover all the ground, whereas UAV communications mainly focus on providing access services to support BSs in the hotspot areas of the dense networks, i.e., airports or resorts, where most users are located in the lounge [19]. Based on the insights of [42], where the serving area can be considered as a regular disc, another strategy considered in this article is the UAV-centric strategy, where paired NOMA users are located inside the coverage disc as shown in Fig. 2, It is also worth noting that the locations of UAVs are properly selected to serve terrestrial users in the hotspot areas based on user density in the UAV-centric strategy. Based on the insights of poisson cluster process (PCP), the users are located in multiple small clusters in practice.

For the UAV-centric strategy, a UAV is located at the original point, which becomes the typical UAV serving users in the typical cell. Therefore, it is assumed that the distance between the UAV at the origin and the nearest UAV is $R$, and the potential paired NOMA users are located in the coverage area within the radius $R / 2$. In the UAV-centric strategy, user pairing strategy is determined by the connected UAV, where all the users in the coverage disc are connected to the UAV. In the user association, for simplicity, we assume that there are two users, near user $w$ and far user $v$, have accessed to the UAV at the origin to perform NOMA. It is assumed that the users are uniformly located, which is according to HPPP, denoted by $\Psi_{u}$ and it is associated 
with the density $\lambda_{u}$, within large ring and small disc with radius $R / 2$ and $R / 4$, respectively. By doing so, NOMA technique can be performed without accurate CSI.

\section{A. SINR Analysis}

For the UAV-centric strategy, the distances between the interfering UAVs and the users are more complicated. For notational simplicity, the location of the $j$-th interfering UAV is denoted by $y_{j}$, where $y_{j} \in \Psi$. The locations of the users are conditioned on the locations of their cluster heads (UAVs). As such, the SINR of the far user $v$ can be derived as

$$
S I N R_{v}=\frac{\left|h_{v}\right|^{2} d_{v}^{-\alpha} P_{u} \alpha_{v}^{2}}{\sigma^{2}+\left|h_{v}\right|^{2} d_{v}^{-\alpha} P_{u} \alpha_{w}^{2}+\sum_{j \in \Psi}\left|g_{j}\right|^{2} P_{u} d_{j}^{-\alpha_{I}}},
$$

where $\left|h_{v}\right|^{2}$ and $d_{v}$ denote the small scale fading coefficient and the distance between the far user and the UAV, $\left|g_{j}\right|^{2}$ and $d_{j}$ denote the small scale fading coefficient and the distance between $j$-th interfering UAV and the user, respectively.

The near user $w$ will first decode the signal of the far user $v$ with the following SINR

$$
S I N R_{w \rightarrow v}=\frac{\left|h_{w}\right|^{2} d_{w}^{-\alpha} P_{u} \alpha_{v}^{2}}{\sigma^{2}+\beta\left|h_{w}\right|^{2} d_{w}^{-\alpha} P_{u} \alpha_{w}^{2}+\sum_{j \in \Psi}\left|g_{j}\right|^{2} P_{u} d_{j}^{-\alpha_{I}}}
$$

where $\left|h_{w}\right|^{2}$ and $d_{w}$ denote the small scale fading coefficient and the distance between the near user and the UAV. If the signal of the $v$-th user can be decoded successfully, the $w$-th user then decodes its own signal. As such, the SINR at the $w$-th user can be expressed as

$$
S I N R_{w}=\frac{\left|h_{w}\right|^{2} d_{w}^{-\alpha} P_{u} \alpha_{w}^{2}}{\sigma^{2}+\beta\left|h_{w}\right|^{2} d_{w}^{-\alpha} P_{u} \alpha_{v}^{2}+\sum_{j \in \Psi}\left|g_{j}\right|^{2} P_{u} d_{j}^{-\alpha_{I}}}
$$

\section{B. Coverage Probability of the UAV-centric Strategy}

Consider a disk centered at the origin with the radius $R / 2$, which has shown in Fig. 2, In order to deploy NOMA protocol, we separate the disc to two parts equally, the small disc with radius $R / 4$ and the ring with radius from $R / 4$ to $R / 2$, to serve paired NOMA users. It is assumed that the near users and the far users are located in the small disc and ring, respectively. Focusing on the typical cell, where a UAV is located at the origin, the PDF of distance for the near users conditioned on serving distance $R$, follows

$$
f_{w}(r \mid R)=\frac{32 r}{R^{2}}, 0 \leq r \leq l_{1},
$$


where $l_{1}=\frac{R}{4}$.

The PDF of far users can be obtained by

$$
f_{v}(r \mid R)=\frac{32 r}{3 R^{2}}, l_{1} \leq r \leq l_{2}
$$

where $l_{2}=\frac{R}{2}$.

In order to derive the system performance, the Laplace transform of UAV interferences needs to be derived. We calculate the Laplace transform of inter-cell interference for the paired users in the following Lemma.

Lemma 5. For the UAV-centric strategy, the Laplace transform of interference distribution conditioned on the serving distance $R$ for paired user is given by

$$
\begin{aligned}
\mathcal{L}_{U}(s \mid R) & =\exp \left(-\frac{l_{I}}{R}\left(1-\left(1+\frac{S P_{u}}{m_{I} l_{I}^{\alpha_{I}}}\right)^{-m_{I}}\right)\right) \\
& \times \exp \left(-\frac{2 \pi \lambda}{\alpha_{I}} \sum_{i=1}^{m_{I}}\left(\begin{array}{c}
m_{I} \\
i
\end{array}\right)\left(\frac{s P_{u}}{m_{I}}\right)^{\delta_{I}}(-1)^{\left(\delta_{I}-i\right)} B\left(\frac{-s P_{u} l_{I}^{-\alpha_{I}}}{m_{I}} ; i-\delta_{I}, 1-m_{I}\right)\right) .
\end{aligned}
$$

where $l_{I}=\sqrt{R^{2}+h^{2}}$.

Proof. Please refer to Appendix C.

It is also worth noting that for the NLoS case, the small-scale fading between users and interfering UAVs can be considered as Rayleigh fading. Thus, the Laplace transform can be further obtained in the following Corollary.

Corollary 3. For the NLoS scenario, the Laplace transform of interference distribution conditioned on the serving distance $R$ is given by

$$
\mathcal{L}_{U}(s \mid R)=\exp \left(-\frac{l_{I}}{R}\left(\frac{S P_{u}}{l_{I}^{\alpha_{I}}+S P_{u}}\right)\right) \exp \left(-\frac{2 \pi \lambda P_{u} l_{I}^{2-\alpha_{I}}}{\alpha_{I}\left(1-\delta_{I}\right)}{ }_{2} F_{1}\left(1,1-\delta_{I} ; 2-\delta_{I} ;-s P_{u} l_{I}^{-\alpha_{I}}\right)\right) .
$$

Then, we focus on the coverage behavior of paired NOMA users in the UAV-centric strategy. In the UAV-centric strategy, the coverage probability is more complicated than the user-centric strategy due to the fact that the interfering UAV located at distance $R$ is necessary to evaluate separately. It is assumed that the target rates of user $w$ and user $v$ are $R_{w}$ and $R_{v}$, respectively. Therefore, the coverage probability of the $w$-th user is given in the following Lemma. 
Lemma 6. The closed-form expression of the coverage probability conditioned on serving distance for the near user is expressed as

$$
\begin{aligned}
& P_{c o v, w}(r \mid R)=\sum_{n=0}^{m-1} \sum_{k=0}^{n} \sum_{l=0}^{k} \frac{(-1)^{n} r_{w}^{\alpha n}}{l !(k-l) !(n-k) !} \Theta_{3} \Theta_{4} \Theta_{5} \\
& \times \exp \left(-m M_{w *} \sigma^{2} r_{w}^{\alpha}-\Theta_{1} r_{w}^{\alpha(i+a)}-\frac{m l_{I}}{R}+\Theta_{2} r_{w}^{\alpha U}\right) r_{w}^{\alpha(1-j) q_{j}+\alpha(i+a-g) q_{g}+\alpha n+\alpha(U-b) q_{u},}
\end{aligned}
$$

where $M_{w}=\frac{\varepsilon_{w}}{P_{u}\left(\alpha_{w}^{2}-\beta \varepsilon_{w} \alpha_{v}^{2}\right)}, M_{v}=\frac{\varepsilon_{v}}{P_{u}\left(\alpha_{v}^{2}-\varepsilon_{v} \alpha_{w}^{2}\right)}, \varepsilon_{w}=2^{R_{w}}-1, \varepsilon_{v}=2^{R_{v}}-1, M_{w *}=\max \left\{M_{w}, M_{v}\right\}$, $r_{w}=\sqrt{r^{2}+h^{2}}, \Theta_{1}=\pi m \delta_{I} \lambda \sum_{i=1}^{m_{I}}\left(\begin{array}{c}m_{I} \\ i\end{array}\right)(-1)^{\delta_{I}-1} \sum_{a=0}^{\infty} \frac{\left(m_{I}\right)_{a}}{a !\left(i-\delta_{I}+a\right)}\left(\frac{M_{w *} P_{u}}{m_{I}}\right)^{i+a} l_{I}^{-\alpha_{I}\left(i-\delta_{I}+a\right)}$,

$\Theta_{2}=\frac{m l_{I}}{R} \sum_{U=0}^{\infty}(-1)^{U} C_{m_{I}+U+1}^{U}\left(\frac{M_{w *} P_{u}}{l_{I}^{\alpha_{I}} m_{I}}\right)^{U}, \Theta_{3}=\sum(n-k) ! \prod_{j=1}^{n-k} \frac{\left(\left(-m M_{w *} \sigma^{2}\right) \prod_{p=0}^{j-1}(1-p)\right)^{q_{j}}}{q_{j} !(j !)^{q_{j}}}$,

$\Theta_{4}=\sum(k-l) ! \prod_{b=1}^{k-l} \frac{\left(\left(-\Theta_{2}\right) \prod_{p=0}^{b-1}(U-p)\right)^{q_{u}}}{q_{u} !(j !)^{q_{u}}}$, and $\Theta_{5}=\sum l ! \prod_{g=1}^{l} \frac{\left(\left(-\Theta_{1}\right) \prod_{p=0}^{g-1}(i+a-g)\right)^{q_{g}}}{q_{g} !(j !)^{q_{g}}}$.

Proof. Please refer to Appendix D.

Similar to Lemma 6, the coverage probability of the far user can be derived in the following Lemma.

Lemma 7. The closed-form expression of the coverage probability conditioned on serving distance for the far user is expressed as

$$
\begin{aligned}
& P_{c o v, v}(r \mid R)=\sum_{n=0}^{m-1} \sum_{k=0}^{n} \sum_{l=0}^{k} \frac{(-1)^{n} r_{v}^{\alpha n}}{l !(k-l) !(n-k) !} \Theta_{3, v} \Theta_{4, v} \Theta_{5, v} \\
& \times \exp \left(-m M_{v} \sigma^{2} r_{v}^{\alpha}-\Theta_{1, v} r_{v}^{\alpha(i+a)}-\frac{m l_{I}}{R}+\Theta_{2, v} r_{v}^{\alpha U}\right) r_{v}^{\alpha(1-j) q_{j}+\alpha(i+a-g) q_{g}+\alpha n+\alpha(U-b) q_{u}},
\end{aligned}
$$

where $r_{v}=\sqrt{r^{2}+h^{2}}, \Theta_{1, v}=\pi m \delta_{I} \lambda \sum_{i=1}^{m_{I}}\left(\begin{array}{c}m_{I} \\ i\end{array}\right)(-1)^{\delta_{I}-1} \sum_{a=0}^{\infty} \frac{\left(m_{I}\right)_{a}}{a !\left(i-\delta_{I}+a\right)}\left(\frac{M_{v} P_{u}}{m_{I}}\right)^{i+a} l_{I}^{-\alpha_{I}\left(i-\delta_{I}+a\right)}$,

$\Theta_{2, v}=\frac{m l_{I}}{R} \sum_{U=0}^{\infty}(-1)^{U} C_{m_{I}+U+1}^{U}\left(\frac{M_{v} P_{u}}{l_{I}^{\alpha} m_{I}}\right)^{U}, \Theta_{3, v}=\sum(n-k) ! \prod_{j=1}^{n-k} \frac{\left(\left(-m M_{v} \sigma^{2}\right) \prod_{p=0}^{j-1}(1-p)\right)^{q_{j}}}{q_{j} !(j !)^{q_{j}}}$,

$\Theta_{4, v}=\sum(k-l) ! \prod_{b=1}^{k-l} \frac{\left(\left(-\Theta_{2, v}\right) \prod_{p=0}^{b-1}(U-p)\right)^{q_{u}}}{q_{u} !(j !)^{q_{u}}}$, and $\Theta_{5, v}=\sum l ! \prod_{g=1}^{l} \frac{\left(\left(-\Theta_{1, v}\right) \prod_{p=0}^{g-1}(i+a-g)\right)^{q_{g}}}{q_{g} !(j !)^{q_{g}}}$.

Proof. Similar to Appendix D, the derivation in (28) can be readily proved.

Then, the coverage probability of paired NOMA users in the UAV-centric strategy can be derived in the following Theorem. 
Theorem 2. Based on Lemma 6 and Lemma 7, the exact expressions of the coverage probability for the paired NOMA users can be expressed as

$$
P_{c o v, w}=\int_{0}^{\infty} \int_{0}^{l_{1}} P_{c o v, w}(r \mid R) f_{w}(r \mid R) d r f_{r}(R) d R
$$

and

$$
P_{c o v, v}=\int_{0}^{\infty} \int_{l_{1}}^{l_{2}} P_{c o v, v}(r \mid R) f_{v}(r \mid R) d r f_{r}(R) d R
$$

where $l_{1}=\frac{R}{4}, l_{2}=\frac{R}{2}, P_{\text {cov }, w}(r \mid R)$ is given in (27), $P_{\text {cov }, w}(r \mid R)$ is given in (28), $f_{w}(r \mid R)$ is given in (23), $f_{v}(r \mid R)$ is given in (24), and $f_{r}(R)$ is given in (12).

Proof. By utilizing the PDF in (23), the coverage probability of the near user conditioned on the serving distance can be obtained by

$$
P_{c o v, w}(R)=\int_{0}^{l_{1}} P_{c o v, w}(r \mid R) f_{w}(r \mid R) d r,
$$

The overall coverage probability can be derived by the serving distance of UAV assisted cellular networks, which can be expressed as

$$
P_{c o v, w}=\int_{0}^{\infty} P_{c o v, w}(R) f_{r}(R) d R
$$

Plugging (12) into (32), and after some mathematical manipulations, the coverage probability of the near user can be obtained. Thus, the proof is complete.

In order to provide more engineering insights, the coverage probability for the near user in the OMA assisted UAV-centric strategy is also derived in the following Corollary. Similar to Corollary 2, we also use TDMA to illustrate the coverage performance for OMA assisted UAV-centric strategy.

Corollary 4. The coverage probability conditioned on the serving distance of the near user for 
the OMA enhanced UAV-centric strategy is expressed in closed-form as

$$
\begin{aligned}
& P_{c o v, w}^{o}(r \mid R)=\sum_{n=0}^{m-1} \sum_{k=0}^{n} \sum_{l=0}^{k} \frac{(-1)^{n} r_{w}^{\alpha n}}{l !(k-l) !(n-k) !} \Theta_{3}^{o} \Theta_{4}^{o} \Theta_{5}^{o} \\
& \times \exp \left(-m M_{w}^{o} \sigma^{2} r_{w}^{\alpha}-\Theta_{1} r_{w}^{\alpha(i+a)}-\frac{m l_{I}}{R}+\Theta_{2} r_{w}^{\alpha U}\right) r_{w}^{\alpha(1-j) q_{j}+\alpha(i+a-g) q_{g}+\alpha n+\alpha(U-b) q_{u}},
\end{aligned}
$$

where $M_{w}^{o}=\frac{\varepsilon_{w}^{o}}{P_{u}}, \varepsilon_{w}^{o}=2^{2 R_{w}}-1, \Theta_{1}^{o}=\pi m \delta_{I} \lambda \sum_{i=1}^{m_{I}}\left(\begin{array}{c}m_{I} \\ i\end{array}\right)(-1)^{\delta_{I}-1} \sum_{a=0}^{\infty} \frac{\left(m_{I}\right)_{a}}{a !\left(i-\delta_{I}+a\right)}\left(\frac{M_{w}^{o} P_{u}}{m_{I}}\right)^{i+a} l_{I}^{-\alpha_{I}\left(i-\delta_{I}+a\right)}$,

$\Theta_{2}^{o}=\frac{m l_{I}}{R} \sum_{U=0}^{\infty}(-1)^{U} C_{m_{I}+U+1}^{U}\left(\frac{M_{w}^{o} P_{u}}{l_{I}^{\alpha} m_{I}}\right)^{U}, \Theta_{3}^{o}=\sum(n-k) ! \prod_{j=1}^{n-k} \frac{\left(\left(-m M_{w}^{o} \sigma^{2}\right) \prod_{p=0}^{j-1}(1-p)\right)^{q_{j}}}{q_{j} !(j !)^{q_{j}}}$,

$\Theta_{4}^{o}=\sum(k-l) ! \prod_{b=1}^{k-l} \frac{\left(\left(-\Theta_{2}^{o}\right) \prod_{p=0}^{b-1}(U-p)\right)^{q_{u}}}{q_{u} !(j !)^{q_{u}}}$, and $\Theta_{5}^{o}=\sum l ! \prod_{g=1}^{l} \frac{\left(\left(-\Theta_{1}^{o}\right) \prod_{p=0}^{g-1}(i+a-g)\right)^{q_{g}}}{q_{g} !(j !)^{q_{g}}}$.

Proof. Following the similar procedure in Appendix D, with interchanging $M_{w}^{f}$ with $M_{w}^{o}$, we can obtain the desired result in (33). Thus, the proof is complete.

\section{NUMERICAL STUdiES}

In this section, numerical results are provided to facilitate the performance evaluation of NOMA assisted UAV cellular networks. Monte Carlo simulations are conducted to verify analytical results. In the considered network, it is assumed that the power allocation factors are $\alpha_{v}^{2}=0.6$ for the far user and $\alpha_{w}^{2}=0.4$ for the near user. The path loss exponent of interference links $\alpha_{I}$ is set to 4 , and the path loss exponent of the desired transmission is smaller than 4 . The height of the UAV is fixed to 100 meters. In Monte Carlo simulations, it is not possible to simulate a real infinite distribution for UAVs. Hence, the UAVs are distributed in a disc, and the radius of the disc is $10000 \mathrm{~m}$. The bandwidth of the downlink transmission is set as $B W=300$ $\mathrm{kHz}$, and the power of AWGN noise is set as $\sigma^{2}=-174+10 \log _{10}(B W) \mathrm{dBm}$. The UAV density $\lambda=\frac{1}{500^{2} \pi}$. It is also worth noting that LoS and NLoS scenarios are indicated by the Nakagami fading parameter $m$, where $m=1$ for NLoS scenarios (Rayleigh fading) and $m>1$ for LoS scenarios. Without loss of generality, we use $m=2$ to represent LoS scenario in Section IV.

\section{A. User-centric strategy}

First, we evaluate the coverage performance of downlink NOMA users in the user-centric strategy. In Fig. 3(a), for a given set of the distance of fixed users, the solid curves and dashed 

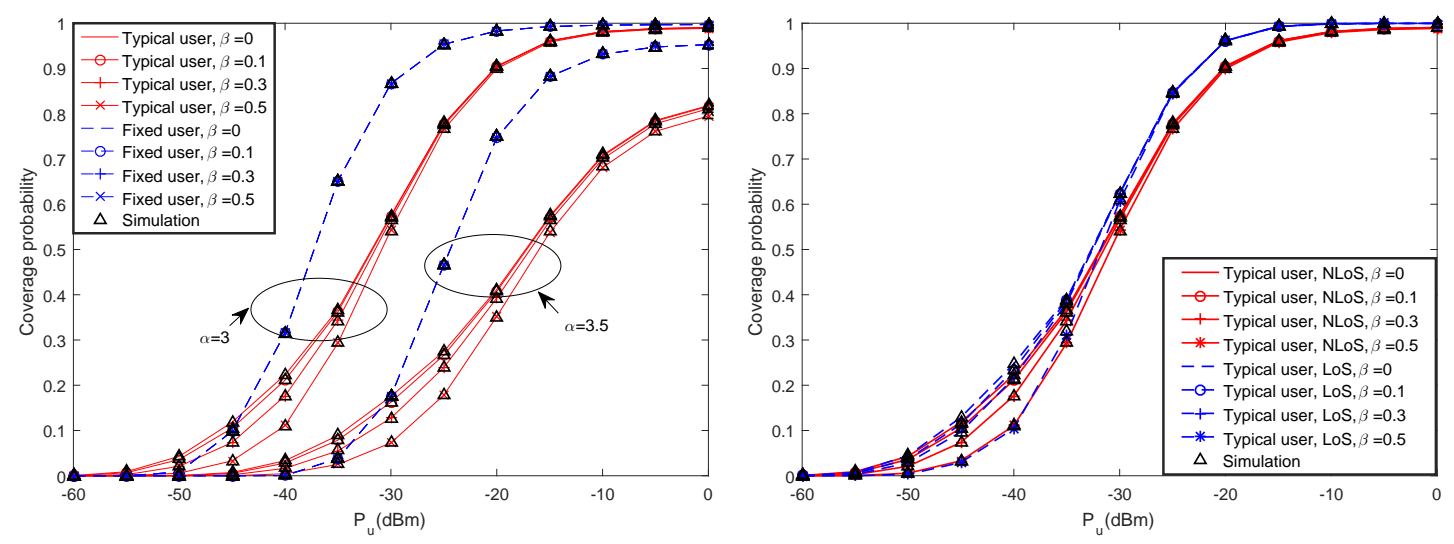

(a) Coverage probability of user-centric NOMA versus (b) Coverage probability of user-centric NOMA versus transmit power in NLoS scenario with different path loss transmit power in both NLoS and LoS scenarios, where exponent, where the fading parameters $m=1$ and $m_{I}=$ the fading parameters $m=2$ and $m_{I}=1$. The path loss 1.

exponent of desire link is set to be $\alpha=3$.

Fig. 3: Coverage probability of paired NOMA users versus the power of UAV in the user-centric strategy, with target rate $R_{t}=1 \mathrm{BPCU}$ and $R_{f}=0.5 \mathrm{BPCU}$. The horizontal distance of the fixed user is $300 \mathrm{~m}$. The exact results of NOMA are calculated from (18).

curves are the coverage probability for typical users and fixed users, respectively. We can see that, as the power of UAV increases, the coverage ceilings, which are the maximum coverage probability for the proposed networks, of both typical users and fixed NOMA users occur. This is due to the fact that, as the higher power level of interfering UAVs is deployed, the received SINR decreases dramatically. It is observed that as imperfect SIC coefficient $\beta$ increases, the coverage probability of typical users decreases, which indicates that the performance of NOMA assisted UAV communication can be effectively improved by decreasing the imperfect SIC coefficient. For example, in the case of $\beta=\frac{2}{3}$, the power residual from imperfect SIC is greater than the power of near users, i.e., $\alpha_{w}^{2}<\alpha_{v}^{2} \beta$. We can also see that in the case of $\beta=0,0.1,0.3,0.5$, the coverage probabilities of fixed users are the same. This is due to the fact that the imperfect SIC is the critical component of typical users, whereas the imperfect SIC has no effect for fixed users in the case $R_{f}=0.5$ bits per channel use (BPCU). As we can see in the figure, the outage of typical users occurs more frequently than fixed users. This is due to the fact that the choice of power allocation factors and the distance of fixed users. Note that the simulation results and analytical results match perfectly in Fig. 3(a), which demonstrate the accuracy of the developed analytical results.

Fig. 3(b) shows the coverage probability achieved by typical users in both NLoS and LoS scenario. In order to better illustrate the performance affected by the LoS transmission, the NLoS case is also shown in the figure as a benchmark for comparison. In Fig. 3(b), we can see that 


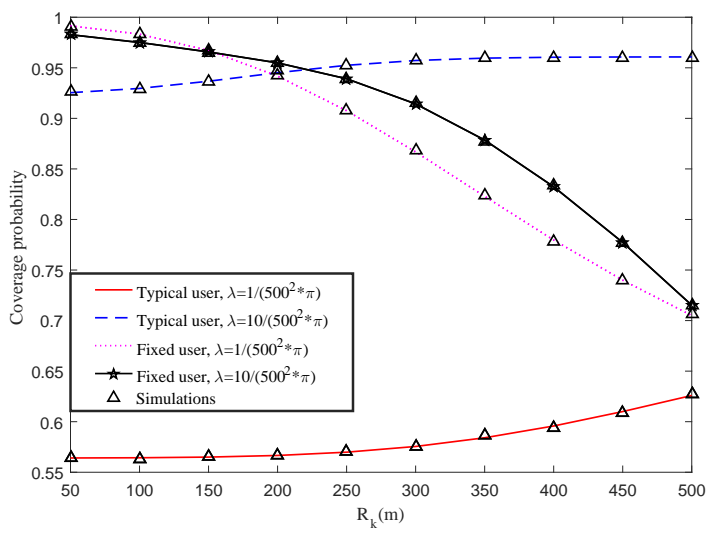

Fig. 4: Coverage probability of user-centric NOMA versus the distance of fixed users, with target rate $R_{t}=1 \mathrm{BPCU}$ and $R_{f}=0.5 \mathrm{BPCU}$. The path loss exponent is fixed to $\alpha=3$, and the power of UAV is fixed to $-30 \mathrm{dBm}$.

higher fading parameter $m$ would result in reduced outage probability for different UAV power levels and different imperfect SIC coefficients. This is because that the LoS link between the UAV and users provides higher received power level. It is also worth noting that for the UAV cellular networks, the proposed network is not in need of a larger UAV power for increasing the coverage probability due to the fact that the coverage ceiling occurs in the high SNR regime.

In Fig. 4, the impact of different choices of UAV density and the distance of fixed users is studied. As can be observed from the figure, increasing the distance of fixed users will decrease the coverage probability for fixed users, whereas the coverage probability of typical users increases. This is due to the fact that the distance of fixed users has affect on user association for typical users. For fixed users, the received power decreases dramatically when the distance increases. On the other hand, for the dashed curve and star curve, where the density of UAV is 10 times greater than the solid curve and dotted curves, the coverage probability of typical NOMA users in the case of high UAV density is much greater than the case of low UAV density. This is because that the number of UAVs is increased, which leads to the decrease of the distance of connected UAV. It is also worth noting that there are two crosses of fixed users, which mean that there exists an optimal distance of fixed users for the given UAV density.

Next, Fig. 5 plots the coverage probability of paired NOMA users in the user-centric strategy versus target rate $R$ and power allocation factor $\alpha_{v}$. It is observed that the coverage probability is zero in the case of inappropriate target rates and power allocation factors, which verifies the insights from Remark 2. The coverage probability of typical users in OMA is also plotted, which indicates that NOMA is capable for outperforming OMA for the appropriate power 


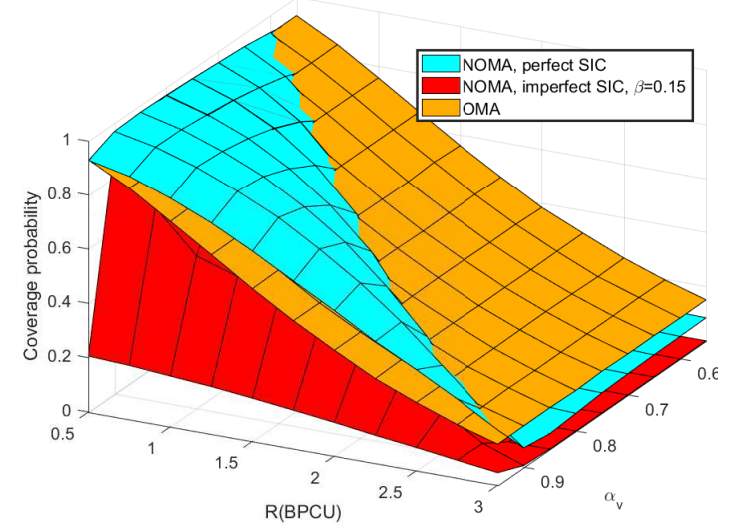

Fig. 5: Coverage probability of typical users versus targeted rate $R_{t}=R$ BPCU, and power allocation factor $\alpha_{v}$, with the imperfect SIC coefficient $\beta=0,0.15$. The target rate of fixed users $R_{f}=0.5 \mathrm{BPCU}$, and the horizontal distance of the fixed user is $300 \mathrm{~m}$. The transmit power of UAVs is fixed to $-30 \mathrm{dBm}$ with path loss exponent $\alpha=3$. The fading parameters $m=3$ and $m_{I}=2$.

allocation factors and target rates of paired users. One can also observe that NOMA cannot outperform OMA in the case of $\beta=0.15$ for the user-centric strategy. This indicates that hybrid NOMA/OMA assisted UAV framework may be a good solution in the case of poor SIC quality. The UAV could intelligently choose the access techniques for improving the system coverage probability.

\section{B. UAV-centric strategy}

In the UAV-centric strategy, $\varepsilon=0.1 \mathrm{~m}$ to evaluate the interference received from the UAV located at the distance $R$. Then, we evaluate the performance of the downlink users in the UAVcentric strategy. In Figs. 6(a) and 6(b), the impact of the NOMA assisted UAV-centric strategy in terms of the coverage probability is studied. The target rates of near users and far users are set as $R_{w}=1.5 \mathrm{BPCU}$ and $R_{v}=1 \mathrm{BPCU}$, respectively. Solid curves and dashed curve are the coverage probability of near users and far users, respectively. An interesting phenomenon occurs in the UAV-centric strategy that in the case $\beta=0.5$, the coverage probability of near users is all zero, which indicates that the transmission is failed. This is again due to the fact that $\alpha_{w}^{2}-\beta \alpha_{v}^{2} \varepsilon_{w}<0$, which verifies our obtained insights in Remark 2 .

Comparing Fig. 6(a) with Fig. 6(b), one can observe that the impact of fading parameter $m$ on the coverage probability is also significant, which is due to the fact that the received power level is greater in the case of larger $m$. Again, we can see that the coverage probability is also one of near users in the case of $\beta=0.5$, which indicates that the LoS propagation has no effect 


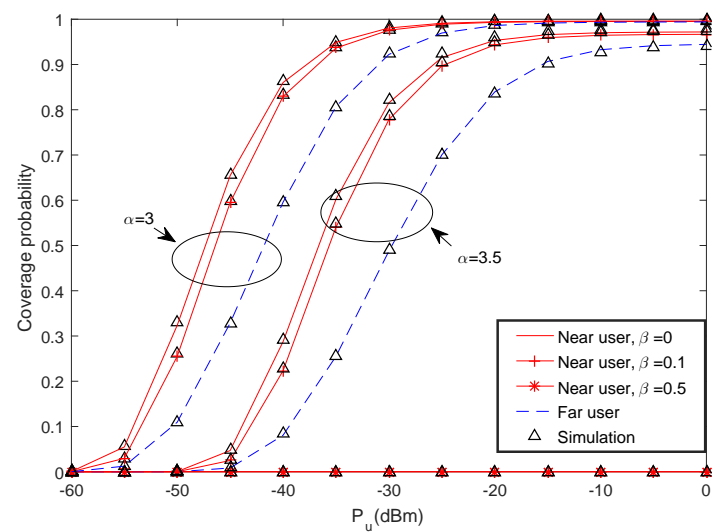

(a) Coverage probability of the UAV-centric NOMA (b) Coverage probability of the UAV-centric NOMA verversus the transmit power in NLoS scenario, where the sus the transmit power in both NLoS and LoS scenarios fading parameters $m=1, m_{I}=1$.

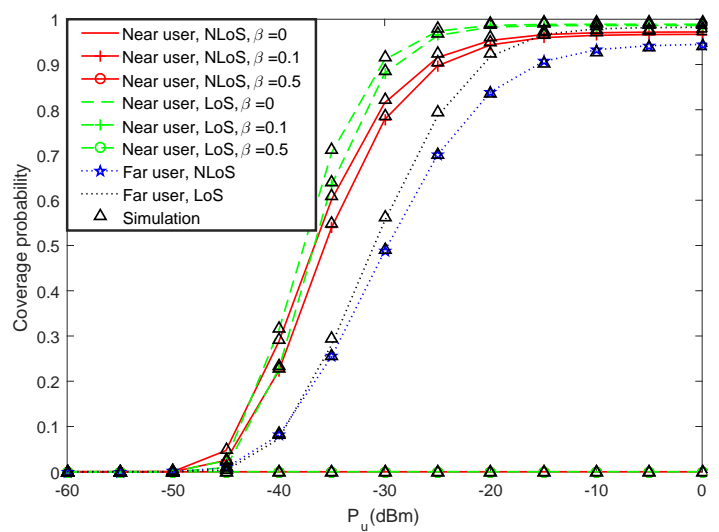

with path loss exponent $\alpha=3.5$, where the fading parameters $m=2, m_{I}=1$.

Fig. 6: Coverage probability of paired NOMA users versus the transmit power, with target rate $R_{w}=1.5$ and $R_{v}=1$ BPCU, respectively. The exact results of NOMA are calculated from (29).

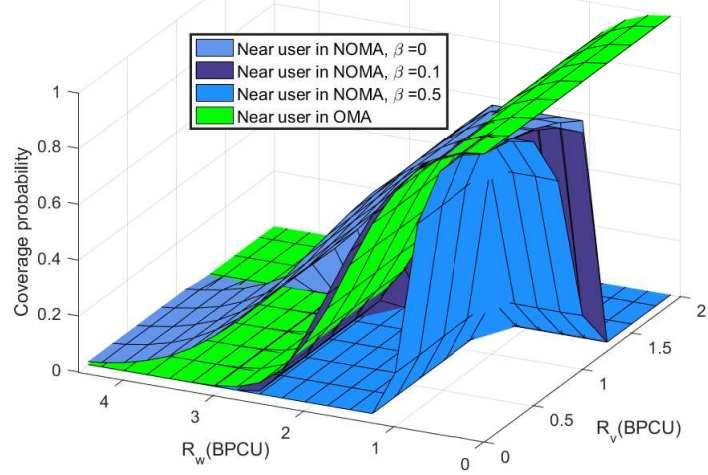

Fig. 7: Coverage probability of the near user versus the target rate. The transmit power of UAVs is fixed to $-40 \mathrm{dBm}$. The fading parameters $m=3$ and $m_{I}=2$.

on Remark 2. It is also worth noting that the coverage probability of the user-centric strategy is much greater than the UAV-centric strategy in the case of $\beta=0.5$, which indicates that the UAV-centric strategy is more susceptible to ipSIC factor than the user-centric strategy.

Fig. 7 plots the coverage probability for near users in the UAV-centric strategy in the cases of $\beta=0, \beta=0.1$, and $\beta=0.5$. One can obtain that on the one hand, inappropriate power allocation will lead to the coverage probability always being zero, which also verified Remark 2 , On the other hand, we can see that in the case of $\beta>0$, the coverage probability decreases dramatically when increasing target rate, which verified that the SIC residue is the dominant interference in NOMA. In order to provide more insights, the coverage performance of OMA in the UAV-centric strategy is also provided. We can see that in the case of $\beta=0$, NOMA performs 
better than OMA, which indicates that the proposed frameworks are analytically shown to be applicable for UAV communications. We can also see that in the case of $\beta=0.1$, the coverage performance of NOMA and OMA assisted UAV cellular networks show closed agreement, which also indicates that hybrid NOMA/OMA assisted UAV framework may be a good solution for the UAV-centric strategy.

\section{CONCLUSIONS}

In this article, we first proposed an overview on a pair of important new paradigms in UAV assisted cellular communications, namely, user-centric strategy and UAV-centric strategy. The user-centric strategy is applicable in the case when all the users located in the Voronoi cell are needed to be served by the UAV simultaneously. The derived results provide the benchmark for the NOMA assisted UAV cellular networks. The UAV-centric strategy is motivated by the fact that, in practice, it is more applicable to serve users in the dense networks. The key idea of the UAV-centric strategy is to provide services for the hotspot areas only, i.e., airports or resorts. Then, the performance of proposed framework were evaluated, where multiple UAVs are distributed in the sky to serve multiple users on the terrestrial. Additionally, new

analytical expressions for interference and coverage probability were derived for characterizing the performance in NOMA assisted UAV cellular frameworks. An important future direction is to extend the 3-D distribution of interference sources to include other interfering UAVs located on the different heights.

\section{Appendix A: Proof of Lemma 2}

Consider a HPPP $\Psi$ with density $\lambda$, the Laplace transform of the interference for the typical user can be expressed as follows:

$$
\mathcal{L}_{t}(s)=\mathbb{E}\left\{\exp \left(-s I_{t, \Psi}\right)\right\}=\mathbb{E}\left\{\exp \left(-s \sum_{j \in \Psi, d_{j}>r_{t}}\left|g_{j}\right|^{2} \frac{P_{u}}{m_{I}} d_{j}^{-\alpha_{I}}\right)\right\} .
$$

Using the moment generating function (MGF) of Gamma random variable $\left|g_{j}\right|$, the Laplace transform can be rewritten to

$$
\mathcal{L}_{t}(s)=\exp \left(-2 \pi \lambda \int_{r_{t}}^{\infty}\left(1-\mathbb{E}_{g}\left\{\exp \left(-s\left|g_{j}\right|^{2} \frac{P_{u}}{m_{I}} r^{-\alpha_{I}}\right)\right\}\right) r d r\right)
$$


With the aid of Laplace transform for the Nakagami-m distribution with fading parameter $m_{I}$, we can obtain $\mathbb{E}_{g}\left\{s\left|g_{j}\right|^{2} \frac{P_{u}}{m_{I}} r^{-\alpha_{I}}\right\}=\left(1+\frac{s P_{u} r^{-\alpha_{I}}}{m_{I}}\right)^{-m_{I}}$. As such, by applying binomial expansion, the Laplace transform of the interference at the typical user can be rewritten to

$$
\begin{aligned}
\mathcal{L}_{t}(s) & =\exp \left(-2 \pi \lambda \int_{r_{t}}^{\infty}\left(1-\left(1+\frac{s P_{u} r^{-\alpha_{I}}}{m_{I}}\right)^{-m_{I}}\right) r d r\right) \\
& =\exp \left(-2 \pi \lambda \int_{r_{t}}^{\infty} \frac{\sum_{i=0}^{m_{I}}\left(\begin{array}{c}
m_{I} \\
i
\end{array}\right)\left(\frac{s P_{u}}{r^{\alpha} m_{I}}\right)^{i}-1}{\left(1+\frac{s P_{u} r^{-\alpha_{I}}}{m_{I}}\right)^{m_{I}}} r d r\right) .
\end{aligned}
$$

Then, after some algebraic manipulations, we have

$$
\begin{aligned}
& \mathcal{L}_{t}(s)=\exp \left(-2 \pi \lambda \sum_{i=1}^{m_{I}}\left(\begin{array}{c}
m_{I} \\
i
\end{array}\right)\left(\frac{s P_{u}}{m_{I}}\right)^{i} \int_{r_{t}}^{\infty} \frac{r^{-\alpha_{I} i+1}}{\left(1+\frac{s P_{u} r^{-\alpha_{I}}}{m_{I}}\right)^{m_{I}}} d r\right) \\
& \underset{=}{(a) \exp }\left(-\frac{2 \pi \lambda}{\alpha_{I}} \sum_{i=1}^{m_{I}}\left(\begin{array}{c}
m_{I} \\
i
\end{array}\right)\left(\frac{s P_{u}}{m_{I}}\right)^{\delta_{I}}(-1)^{\delta_{I}-1} \int_{0}^{-\frac{s P_{u}}{r_{t}^{\alpha I} m_{I}}} \frac{t^{i-\delta_{I}-1}}{(1-t)^{m_{I}}} d t\right) \text {, }
\end{aligned}
$$

where $(a)$ is obtained by using $t=-\frac{s P_{u}}{r^{\alpha} I m_{I}}$. Based on [43, eq. (8.391)], we can finally obtain the Laplace transform of the interference in the user-centric strategy in (13).

\section{Appendix B: Proof of Lemma 3}

Then, we derive the coverage probability of the typical user as

$$
\begin{aligned}
& P_{t, \text { near }}(r)=\mathbb{E}_{I_{\Psi}}\left\{\operatorname{Pr}\left(\left|h_{w}\right|^{2}<M_{t *}\left(\sigma^{2}+I_{\Psi}\right) r_{t}^{\alpha}\right)\right\} \\
& =\exp \left(-m M_{t *} \sigma^{2} r_{t}^{\alpha}\right) \mathbb{E}_{I_{\Psi}}\left\{\exp \left(-m M_{t *} I_{\Psi} r_{t}^{\alpha}\right)\right\} \sum_{n=0}^{m-1} \frac{\left(m M_{t *}\left(\sigma^{2}+I_{\Psi}\right) r_{t}^{\alpha}\right)^{n}}{n !} \\
& =\sum_{n=0}^{m-1} \sum_{p=0}^{n}\left(\begin{array}{c}
n \\
p
\end{array}\right) \frac{r_{t}^{\alpha n}(-1)^{n}}{n !} \underbrace{\exp \left(-m M_{t *} \sigma^{2} r_{t}^{\alpha}\right)\left(-m M_{t *} \sigma^{2}\right)^{p}}_{\Lambda_{1}} \underbrace{\mathbb{E}_{I_{\Psi}}\left\{\exp \left(-m M_{t *} I_{\Psi} r_{t}^{\alpha}\right)\right\}\left(-m M_{t} I_{\Psi}\right)^{n-p}}_{\Lambda_{2}} .
\end{aligned}
$$

Using the fact that

$$
\left.\frac{d^{p}\left(\exp \left(-m M_{t *} \sigma^{2} y\right)\right)}{d y^{p}}\right|_{y=r_{t}^{\alpha}}=\exp \left(-m M_{t *} \sigma^{2} r_{t}^{\alpha}\right)\left(-m M_{t *} \sigma^{2}\right)^{p}
$$


we can have

$$
\Lambda_{1}=\left.\frac{d^{p}\left(\exp \left(-m M_{t *} \sigma^{2} y\right)\right)}{d y^{p}}\right|_{y=r_{t}^{\alpha}}
$$

Now, we apply the Fa à di Bruno's formula to solve the derivative of $p$-th order as follows:

$$
\Lambda_{1}=\exp \left(-m M_{t *} \sigma^{2} r_{t}^{\alpha}\right) \sum p ! \prod_{j=1}^{p} \frac{\left(\left(-m M_{t *} \sigma^{2}\right) \prod_{k=0}^{j-1}(1-k) r_{t}^{\alpha(1-j)}\right)^{q_{j}}}{q_{j} !(j !)^{q_{j}}}
$$

where the sum $q_{j}$ is over all p-tuples of nonnegative integers satisfying the constraint

$$
1 \cdot q_{1}+2 \cdot q_{2}+\cdots+p \cdot q_{p}=p
$$

Similar to the steps from (B.2) to (B.4), $\Lambda_{2}$ can be expressed to

$$
\begin{aligned}
& \Lambda_{2}=\mathbb{E}_{I_{\Psi}}\left\{\exp \left(-m M_{t *} I_{\Psi} r_{t}^{\alpha}\right)\left(-m M_{t *} I_{\Psi}\right)^{n-p}\right\} \\
& =\mathbb{E}_{I_{\Psi}}\left\{\left.\frac{d^{n-p}\left(\exp \left(-m M_{t *} I_{\Psi} y\right)\right)}{d y^{n-p}}\right|_{y=r_{t}^{\alpha}}\right\} \\
& =\left.\frac{d^{n-p}\left(m \mathcal{L}_{t}\left(M_{t *} y\right)\right)}{d y^{n-p}}\right|_{y=r_{t}^{\alpha}} .
\end{aligned}
$$

It is challenging to derive $(n-p)$-th order derivation of incomplete Beta function directly. Thus, the derived incomplete Beta function in (13) can be written to

$$
\begin{aligned}
B\left(\frac{-s P_{u}}{m_{I} r_{t}^{\alpha_{I}}} ; i-\delta_{I}, 1-m_{I}\right) & =\left(\frac{-s P_{u}}{m_{I} r_{t}^{\alpha_{I}}}\right)^{\left(i-\delta_{I}\right)} \frac{1}{i-\delta_{I}}{ }_{2} F_{1}\left(1-\delta_{I}, m_{I} ; 2-\delta_{I} ;\left(\frac{-s P_{u}}{m_{I} r_{t}^{\alpha_{I}}}\right)\right) \\
& =\left(\frac{-s P_{u}}{m_{I} r_{t}^{\alpha_{I}}}\right)^{\left(i-\delta_{I}+a\right)} \sum_{a=0}^{\infty} \frac{\left(m_{I}\right)_{a}}{a !\left(i-\delta_{I}+a\right)},
\end{aligned}
$$

where $\left(m_{I}\right)_{a}$ denotes rising Pochhammer symbol, which can be calculated as $\frac{\Gamma\left(m_{I}+a\right)}{\Gamma\left(m_{I}\right)}$.

Thus, the Laplace transform can be rewritten to

$$
\mathcal{L}_{t}(s)=\exp \left(-\frac{2 \pi \lambda}{\alpha_{I}} \sum_{a=0}^{\infty} \frac{\left(m_{I}\right)_{a}}{a !\left(i-\delta_{I}+a\right)} \sum_{i=1}^{m_{I}}\left(\begin{array}{c}
m_{I} \\
i
\end{array}\right)\left(\frac{s P_{u}}{m_{I}}\right)^{i+a}(-1)^{a} r_{t}^{-\alpha_{I}\left(i-\delta_{I}+a\right)}\right) .
$$

Then, substituting (B.8) into (B.6), and using Fa à di Bruno's formula, (B.6) can be transformed 
into

$$
\begin{aligned}
& \Lambda_{2}=\left.\frac{d^{n-p}\left(\exp \left(-\Lambda_{3} y^{\delta}\right)\right)}{d y^{n-p}}\right|_{y=r_{t}^{\alpha}} \\
& =\exp \left(-\Lambda_{3} r_{t}^{2+\left(\alpha-\alpha_{I}\right)(i+a)}\right) \sum(n-p) ! \prod_{b=1}^{n-p} \frac{\left(\left(-\Lambda_{3}\right) \prod_{k=0}^{b-1}(\delta-k) r_{t}^{2+\left(\alpha-\alpha_{I}\right)(i+a)-\alpha b}\right)^{q_{b}}}{q_{b} !(b !)^{q_{b}}},
\end{aligned}
$$

where $\Lambda_{3}=\frac{2 \pi \lambda}{\alpha_{I}} \sum_{a=0}^{\infty} \frac{\left(m_{I}\right)_{a}}{a !\left(i-\delta_{I}+a\right)} \sum_{i=1}^{m_{I}}\left(\begin{array}{c}m_{I} \\ i\end{array}\right)\left(\frac{M_{t *} P_{u}}{m_{I}}\right)^{i+a}(-1)^{a}$, and $q_{b}$ is over all $(n-p)$-tuples of nonnegative integers satisfying the constraint $1 \cdot q_{1}+2 \cdot q_{2}+\cdots+(n-p) \cdot q_{b}=(n-p)$.

Substituting (B.4) and (B.9) into (B.1), we can derive the coverage probability conditioned on the distance for the typical user in the near user case, as given in (16). The proof is complete.

\section{Appendix C: PROOF OF Lemma 5}

Unlike the user-centric strategy, the interfering UAV located at the distance $R$ is necessary to evaluate separately in the UAV-centric strategy. In this section, we evaluate the Laplace transform of inter-cell interference of the near user in the UAV-centric strategy, where the intercell interference experience at the near user can be given by

$$
I_{w, \Psi}=\underbrace{\sum_{j \in \Psi, d_{j}>R}\left|g_{j}\right|^{2} P_{u} d_{j}^{-\alpha_{I}}}_{I_{2}}+\underbrace{\left|g_{1}\right|^{2} P_{u} R^{-\alpha_{I}}}_{I_{1}},
$$

where $I_{1}$ denotes the received power from the interfering UAV located at the distance $R$, and $I_{2}$ denotes the received power from all other interfering UAVs except the one located at the distance $R$. For the near user at the typical cell, the Laplace transform of interference power distribution conditioned on the serving distance $R$ is given by

$$
\begin{aligned}
\mathcal{L}_{w}(s \mid R) & =\mathbb{E}\left\{\exp \left(-s I_{w, \Psi}\right) \mid R\right\}=\mathbb{E}\left\{\exp \left(-s \sum_{j \in \Psi, d_{j}>R}\left|g_{j}\right|^{2} \frac{P_{u}}{m_{I}} d_{j}^{-\alpha_{I}}-s\left|g_{1}\right|^{2} \frac{P_{u}}{m_{I}} R^{-\alpha_{I}}\right) \mid R\right\} \\
& =\mathbb{E}\left\{\exp \left(-s \sum_{j \in \Psi, d_{j}>R} \mathbb{E}_{g}\left\{\left|g_{j}\right|^{2}\right\} \frac{P_{u}}{m_{I}} d_{j}^{-\alpha_{I}}-s \mathbb{E}_{g}\left\{\left|g_{1}\right|^{2}\right\} \frac{P_{u}}{m_{I}} R^{-\alpha_{I}}\right) \mid R\right\} .
\end{aligned}
$$

We first evaluate the Laplace transform of $I_{2}$. Using the MGF of Gamma random variable 
$\left|g_{j}\right|$, and after some algebraic manipulations, $I_{2}$ can be rewritten to

$$
I_{2}=\exp \left(-2 \pi \lambda \int_{l_{I}}^{\infty}\left(1-\mathbb{E}_{g}\left\{\exp \left(-s\left|g_{j}\right|^{2} \frac{P_{u}}{m_{I}} r^{-\alpha_{I}}\right)\right\}\right) r d r\right)
$$

where $l_{I}=\sqrt{R^{2}+h^{2}}$.

Similar to the arguments from (A.2) to (A.4), the Laplace transform of $I_{2}$ can be obtained as

$$
I_{2}=\exp \left(-\frac{2 \pi \lambda}{\alpha_{I}} \sum_{i=1}^{m_{I}}\left(\begin{array}{c}
m_{I} \\
i
\end{array}\right)\left(\frac{s P_{u}}{m_{I}}\right)^{\delta_{I}}(-1)^{\left(\delta_{I}-i\right)} B\left(\frac{-s P_{u}}{l_{I}^{\alpha_{I}} m_{I}} ; i-\delta_{I}, 1-m_{I}\right)\right) .
$$

Note that the MGF derived in (C.4) does not include the interfering UAV located at the distance $R$ strictly, which is actually the largest interference source. Therefore, using Poisson Hole Process (PHP), the first interference located at distance $R$ can be derived as follows:

$$
I_{1}=\exp \left(\frac{-2 \pi}{\pi(R+\varepsilon)^{2}-\pi(R-\varepsilon)^{2}} \int_{l_{I}-\varepsilon}^{l_{I}+\varepsilon}\left(1-\mathbb{E}_{g}\left\{\exp \left(-s\left|g_{1}\right|^{2} \frac{P_{u}}{m_{I}} l_{I}^{-\alpha_{I}}\right)\right\}\right) r d r\right)
$$

where $\varepsilon$ is a small distance to evaluate the first interfering UAV.

With the aid of Laplace transform for the Nakagami- $m$ distribution with fading parameter $m_{I}$, we can obtain $\mathbb{E}_{g}\left\{\left|g_{1}\right|^{2} \frac{P_{u}}{m_{I}} d_{1}^{-\alpha_{I}}\right\}=\left(1+\frac{s P_{u} l_{I}^{-\alpha_{I}}}{m_{I}}\right)^{-m_{I}}$. As such, the Laplace transform of the first interfering UAV can be rewritten to

$$
\begin{aligned}
I_{1} & =\exp \left(\frac{-1}{2 R \varepsilon} \int_{l_{I}-\varepsilon}^{l_{I}+\varepsilon}\left(1-\left(1+\frac{s P_{u}}{m_{I} l_{I}^{\alpha_{I}}}\right)^{-m_{I}}\right) r d r\right) \\
& =\exp \left(-\frac{l_{I}}{R}+\frac{l_{I}}{R}\left(1+\frac{s P_{u}}{l_{I}^{\alpha_{I}} m_{I}}\right)^{-m_{I}}\right) .
\end{aligned}
$$

Based on (C.4) and (C.6), we can obtain the Laplace transform of the near user for the UAV-Centric strategy as given in (25). The proof is complete.

\section{APPENDiX D: ProOf of Lemma 6}

In order to prove the desired result, and according to Newton's Generalization of the binomial theorem [44], we first transform (C.6) into

$$
I_{1}=\exp \left(-\frac{l_{I}}{R}+\frac{l_{I}}{R} \sum_{U=0}^{\infty}(-1)^{U} C_{m_{I}+U+1}^{U}\left(\frac{s P_{u}}{l_{I}^{\alpha} m_{I}}\right)^{U}\right),
$$


where $C_{m_{I}+U+1}^{U}=\frac{\left(m_{I}+U+1\right)\left(m_{I}+U\right)+\cdots\left(m_{I}+2\right)}{k !}$.

According to the SINR expressions in (21) and (22), and similar to Appendix B, we can derive the coverage probability conditioned on the serving distance $R$ of the near user in the UAV-centric strategy to

$$
P_{c o v, w}(r \mid R)=\exp \left(-m M_{w *}\left(\sigma^{2}+I_{1}+I_{2}\right) r_{w}^{\alpha}\right) \sum_{n=0}^{m-1} \frac{\left(m M_{w *}\left(\sigma^{2}+I_{1}+I_{2}\right) r_{w}^{\alpha}\right)^{n}}{n !} .
$$

By applying polynomial expansion to ( $(\underline{D} .2)$, the coverage probability can be rewritten to

$$
\begin{aligned}
P_{c o v, w}(r \mid R) & =\sum_{n=0}^{m-1} \sum_{k=0}^{n} \sum_{l=0}^{k} \frac{(-1)^{n} r_{w}^{\alpha n}}{l !(k-l) !(n-k) !} \exp \left(-m M_{w *} \sigma^{2} r_{w}^{\alpha}\right)\left(-m M_{w *} \sigma^{2}\right)^{n-k} \\
& \times \mathbb{E}_{I_{1}}\left\{\exp \left(-m M_{w *} I_{1} r_{w}^{\alpha}\right)\left(-m M_{w *} I_{1}\right)^{k-l}\right\} \mathbb{E}_{I_{2}}\left\{\exp \left(-m M_{w *} I_{2} r_{w}^{\alpha}\right)\left(-m M_{w *} I_{2}\right)^{l}\right\} .
\end{aligned}
$$

Following the similar steps from (B.1) to (B.9), and according to Fa à di Bruno's formula, we can readily derive that the first interference $I_{1}$ to

$$
\left.\frac{d^{k-l} \mathcal{L}\left(M_{w *} x\right)}{d x^{k-l}}\right|_{x=r_{w}^{\alpha}}=\exp \left(-\frac{l_{I} m}{R}+\Theta_{2} r_{w}^{\alpha U}\right) \sum(k-l) ! \prod_{j=1}^{k-l} \frac{\left(\left(-\Theta_{2}\right) \prod_{p=0}^{j-1}(u-p) r_{w}^{\alpha(U-j)}\right)^{q_{u}}}{q_{u} !(j !)^{q_{u}}},
$$

where $\Theta_{2}=\frac{l_{I} m}{R} \sum_{U=0}^{\infty}(-1)^{U} C_{m_{I}+U+1}^{u}\left(\frac{M_{w *} P_{u}}{l_{I}^{\alpha I} m_{I}}\right)^{U}$. Then, the closed-form expression of the coverage probability for the near user in (27) can be obtained. Thus, the Lemma is proved.

\section{REFERENCES}

[1] T. Hou, Y. Liu, Z. Song, X. Sun, and Y. Chen, "Non-orthogonal multiple access in multi-UAV networks," in The 2019 IEEE 90th Vehicular Technology Conference, Hawaii, USA, Sep. 2019, pp. 1-1.

[2] Y. Zeng, R. Zhang, and T. J. Lim, "Wireless communications with unmanned aerial vehicles: opportunities and challenges," IEEE Commun. Mag., vol. 54, no. 5, pp. 36-42, May 2016.

[3] M. Mozaffari, W. Saad, M. Bennis, and M. Debbah, "Unmanned aerial vehicle with underlaid device-to-device communications: Performance and tradeoffs," IEEE Trans. Wireless Commun., vol. 15, no. 6, pp. 3949-3963, Jun. 2016.

[4] "Study on enhanced LTE support for aerial vehicles (release 15)," Online: ftp://www.3gpp.org/specs/archive/36_series/36.777, vol. 3GPP TR 36.777, Jun. 2017.

[5] Z. Ding, Y. Liu, J. Choi, Q. Sun, M. Elkashlan, C. I, and H. V. Poor, "Application of non-orthogonal multiple access in LTE and 5G networks," IEEE Commun. Mag., vol. 55, no. 2, pp. 185-191, Feb. 2017.

[6] Z. Qin, J. Fan, Y. Liu, Y. Gao, and G. Y. Li, "Sparse representation for wireless communications: A compressive sensing approach," IEEE Signal Process. Mag., vol. 35, no. 3, pp. 40-58, May 2018.

[7] Z. Ding, P. Fan, and H. V. Poor, "Impact of user pairing on 5G nonorthogonal multiple-access downlink transmissions," IEEE Trans. Veh. Technol., vol. 65, no. 8, pp. 6010-6023, Aug. 2016.

[8] M. Shirvanimoghaddam, M. Dohler, and S. J. Johnson, "Massive non-orthogonal multiple access for cellular IoT: Potentials and limitations," IEEE Commun. Mag., vol. 55, no. 9, pp. 55-61, Sep. 2017.

[9] Y. Liu, Z. Qin, M. Elkashlan, Z. Ding, A. Nallanathan, and L. Hanzo, "Nonorthogonal multiple access for 5G and beyond," Proc. of the IEEE, vol. 105, no. 12, pp. 2347-2381, Dec. 2017.

[10] S. M. R. Islam, M. Zeng, O. A. Dobre, and K. Kwak, "Resource allocation for downlink NOMA systems: Key techniques and open issues," IEEE Wireless Commun., vol. 25, no. 2, pp. 40-47, Apr. 2018.

[11] S. M. R. Islam, N. Avazov, O. A. Dobre, and K. Kwak, "Power-domain non-orthogonal multiple access (NOMA) in 5G systems: Potentials and challenges," IEEE Commun. Surveys Tuts., vol. 19, no. 2, pp. 721-742, Secondquarter 2017. 
[12] Z. Qin, X. Yue, Y. Liu, Z. Ding, and A. Nallanathan, "User association and resource allocation in unified non-orthogonal multiple access enabled heterogeneous ultra dense networks," IEEE Commun. Mag., vol. 56, no. 6, pp. 86-92, Jun. 2018.

[13] A. A. Khuwaja, Y. Chen, N. Zhao, M. Alouini, and P. Dobbins, "A survey of channel modeling for UAV communications," IEEE Commun. Surveys Tuts., vol. 20, no. 4, pp. 2804-2821, Fourthquarter 2018.

[14] N. Goddemeier and C. Wietfeld, "Investigation of air-to-air channel characteristics and a UAV specific extension to the Rice model," in 2015 IEEE Globecom Workshops (GC Wkshps), Dec. 2015, pp. 1-5.

[15] F. Jiang and A. L. Swindlehurst, "Optimization of UAV heading for the ground-to-air uplink," IEEE J. Sel. Areas Commun., vol. 30, no. 5, pp. 993-1005, Jun. 2012.

[16] V. V. Chetlur and H. S. Dhillon, "Downlink coverage analysis for a finite 3-D wireless network of unmanned aerial vehicles," IEEE Trans. Commun., vol. 65, no. 10, pp. 4543-4558, Oct. 2017.

[17] A. Goldsmith, Wireless Communication. Cambridge University Press, 2nd ed, 2010.

[18] S. Zhang, Y. Zeng, and R. Zhang, "Cellular-enabled UAV communication: A connectivity-constrained trajectory optimization perspective," IEEE Trans. Commun., vol. 67, no. 3, pp. 2580-2604, Mar. 2019.

[19] J. Lyu, Y. Zeng, and R. Zhang, "UAV-aided offloading for cellular hotspot," IEEE Trans. Wireless Commun., vol. 17, no. 6, pp. 3988-4001, Jun. 2018.

[20] L. Dai, B. Wang, Z. Ding, Z. Wang, S. Chen, and L. Hanzo, "A survey of non-orthogonal multiple access for 5G," IEEE Commun. Surveys Tuts., vol. 20, no. 3, pp. 2294-2323, thirdquarter 2018.

[21] W. Shin, M. Vaezi, B. Lee, D. J. Love, J. Lee, and H. V. Poor, "Non-orthogonal multiple access in multi-cell networks: Theory, performance, and practical challenges," IEEE Commun. Mag., vol. 55, no. 10, pp. 176-183, Oct. 2017.

[22] Z. Ding, Z. Yang, P. Fan, and H. V. Poor, "On the performance of non-orthogonal multiple access in 5G systems with randomly deployed users," IEEE Signal Process. Lett., vol. 21, no. 12, pp. 1501-1505, Dec. 2014.

[23] Y. Liu, Z. Ding, M. Elkashlan, and H. V. Poor, "Cooperative non-orthogonal multiple access with simultaneous wireless information and power transfer," IEEE J. Sel. Areas Commun., vol. 34, no. 4, pp. 938-953, Apr. 2016.

[24] X. Yue, Y. Liu, S. Kang, A. Nallanathan, and Y. Chen, "Modeling and analysis of two-way relay non-orthogonal multiple access systems," IEEE Trans. Commun., vol. 66, no. 9, pp. 3784-3796, Sep. 2018.

[25] T. Hou, X. Sun, and Z. Song, "Outage performance for non-orthogonal multiple access with fixed power allocation over Nakagami-m fading channels," IEEE Commun. Lett., vol. 22, no. 4, pp. 744-747, Apr. 2018.

[26] Y. Mao, B. Clerckx, and V. O. K. Li, "Rate-splitting for downlink multi-user multi-antenna systems: Bridging NOMA and conventional linear precoding," arXiv, vol. 1710.11018v1, pp. 1-1, Aug. 2017.

[27] — "Rate-splitting for multi-antenna non-orthogonal unicast and multicast transmission: Spectral and energy efficiency analysis," arXiv, vol. 1808.08325v1, pp. 1-1, Aug. 2018.

[28] Y. Liu, Z. Qin, M. Elkashlan, Y. Gao, and L. Hanzo, "Enhancing the physical layer security of non-orthogonal multiple access in large-scale networks," IEEE Trans. Wireless Commun., vol. 16, no. 3, pp. 1656-1672, Mar. 2017.

[29] Y. Zeng, J. Xu, and R. Zhang, "Energy minimization for wireless communication with rotary-wing UAV," IEEE Trans. Wireless Commun., vol. 18, no. 4, pp. 2329-2345, Apr. 2019.

[30] Y. Zeng, J. Lyu, and R. Zhang, "Cellular-connected UAV: Potential, challenges, and promising technologies," IEEE Wireless Commun., vol. 26, no. 1, pp. 120-127, Feb. 2019.

[31] Y. Liu, Z. Qin, Y. Cai, Y. Gao, G. Y. Li, and A. Nallanathan, "UAV communications based on non-orthogonal multiple access," IEEE Wireless Commun., vol. 26, no. 1, pp. 52-57, Feb. 2019.

[32] X. Liu, Y. Liu, Y. Chen, and L. Hanzo, "Trajectory design and power control for multi-UAV assisted wireless networks: A machine learning approach,” arXiv, vol. 1812.07665v1, pp. 1-1, Dec. 2018.

[33] N. Zhao, X. Pang, Z. Li, Y. Chen, F. Li, Z. Ding, and M. Alouini, "Joint trajectory and precoding optimization for UAV-assisted NOMA networks," IEEE Trans. Commun., vol. 67, no. 5, pp. 3723-3735, May 2019.

[34] T. M. Nguyen, W. Ajib, and C. Assi, "A novel cooperative NOMA for designing UAV-assisted wireless backhaul networks," IEEE J. Sel. Areas Commun., vol. 36, no. 11, pp. 2497-2507, Nov. 2018.

[35] T. Hou, Y. Liu, Z. Song, X. Sun, and Y. Chen, "Multiple antenna aided NOMA in UAV networks: A stochastic geometry approach," IEEE Trans. Commun., vol. 67, no. 2, pp. 1031-1044, Feb. 2019.

[36] W. Mei and R. Zhang, "Uplink cooperative NOMA for cellular-connected UAV," IEEE J. Sel. Areas Commun., vol. 13, no. 3, pp. 644-656, Jun. 2019.

[37] L. Liu, S. Zhang, and R. Zhang, "Exploiting NOMA for multi-beam UAV communication in cellular uplink," arXiv, vol. 1810.10839v1, pp. 1-1, Oct. 2018.

[38] K. Han, K. Huang, and R. W. Heath, "Connectivity and blockage effects in millimeter-wave air-to-everything networks," IEEE Wireless Commun. Lett., vol. 8, no. 2, pp. 388-391, Apr. 2019.

[39] T. Qi, W. Feng, and Y. Wang, "Outage performance of non-orthogonal multiple access based unmanned aerial vehicles satellite networks," China Communications, vol. 15, no. 5, pp. 1-8, May 2018.

[40] N. Zhao, W. Lu, M. Sheng, Y. Chen, J. Tang, F. R. Yu, and K. Wong, "UAV-assisted emergency networks in disasters," IEEE Wireless Commun., vol. 26, no. 1, pp. 45-51, Feb. 2019.

[41] J. G. Andrews, F. Baccelli, and R. K. Ganti, "A tractable approach to coverage and rate in cellular networks," IEEE Trans. Commun., vol. 59, no. 11, pp. 3122-3134, Nov. 2011.

[42] K. S. Ali, M. Haenggi, H. ElSawy, A. Chaaban, and M. Alouini, "Downlink non-orthogonal multiple access (NOMA) in poisson networks," IEEE Trans. Commun., vol. 67, no. 2, pp. 1613-1628, Feb. 2019.

[43] I. S. Gradshteyn and I. M. Ryzhik, Table of Integrals, Series and Products. New York: Academic Press, 6th ed, 2000. 
[44] N. Bourbaki, Elements of the History of Mathematics Paperback. Springer Berlin Heidelberg, 2nd ed, 2008. 